J. DIFFERENTIAL GEOMETRY

63 (2003) 231-277

\title{
A KAWAMATA-VIEHWEG VANISHING THEOREM ON COMPACT KÄHLER MANIFOLDS
}

\author{
JEAN-PIERRE DEMAILLY \& THOMAS PETERNELL
}

\begin{abstract}
We prove a Kawamata-Viehweg vanishing theorem on a normal compact Kähler space $X$ : if $L$ is a nef line bundle with $L^{2} \neq 0$, then $H^{q}\left(X, K_{X}+L\right)=$ 0 for $q \geq \operatorname{dim} X-1$. As an application we complete a part of the abundance theorem for minimal Kähler threefolds: if $X$ is a minimal Kähler threefold, then the Kodaira dimension $\kappa(X)$ is nonnegative.
\end{abstract}

\section{Introduction}

In this paper we establish the following Kawamata-Viehweg type vanishing theorem on a compact Kähler manifold or, more generally, a normal compact Kähler space:

Theorem 0.1. Let $X$ be a normal compact Kähler space of dimension $n$ and $L$ a nef line bundle on $X$. Assume that $L^{2} \neq 0$. Then

$$
H^{q}\left(X, K_{X}+L\right)=0
$$

for $q \geq n-1$.

In general, one expects a vanishing

$$
H^{q}\left(X, K_{X}+L\right)=0
$$

for $q \geq n+1-\nu(L)$, where $\nu(L)$ is the numerical Kodaira dimension of the nef line bundle $L$, i.e., $\nu(L)$ is the largest integer $\nu$ such that $L^{\nu} \neq 0$.

Received 10/24/2002. 
Of course, when $X$ is projective, Theorem 0.1 is contained in the usual Kawamata-Viehweg vanishing theorem, but the methods of proof in the algebraic case clearly fail in the general Kähler setting. Instead we proceed in the following way: Clearly we may assume that $X$ is smooth and by Serre duality, only the cohomology group $H^{n-1}$ is of interest. Take a singular metric $h$ on $L$ with positive curvature current $T$ with local weight function $h$. By $[29,6]$ there exists a decomposition

$$
T=\sum \lambda_{j} D_{j}+G
$$

where $\lambda_{j} \geq 1$ are irreducible divisors, and $G$ is a pseudo-effective current such that $G \mid D_{i}$ is pseudo-effective for all $i$. Consider the multiplier ideal sheaf $\mathcal{I}(h)$. We associate to $h$ another, "upper regularized" multiplier ideal sheaf $\mathcal{I}_{+}(h)$ by setting

$$
\mathcal{I}_{+}(h):=\lim _{\varepsilon \rightarrow 0_{+}} \mathcal{I}\left(h^{1+\varepsilon}\right)=\lim _{\varepsilon \rightarrow 0_{+}} \mathcal{I}((1+\varepsilon) \varphi) .
$$

It is unknown whether $\mathcal{I}(h)$ and $\mathcal{I}_{+}(h)$ actually differ; in all known examples they are equal. Then in Section 2 the following vanishing theorem is proved:

Theorem 0.2. Let $(L, h)$ be a holomorphic line bundle over a compact Kähler $n$-fold $X$. Assume that $L$ is nef and has numerical Kodaira dimension $\nu(L)=\nu \geqslant 0$, i.e., $c_{1}(L)^{\nu} \neq 0$ and $\nu$ is maximal. Then the morphism

$$
H^{q}\left(X, \mathcal{O}\left(K_{X}+L\right) \otimes \mathcal{I}_{+}(h)\right) \longrightarrow H^{q}\left(X, K_{X}+L\right)
$$

induced by the inclusion $\mathcal{I}_{+}(h) \subset \mathcal{O}_{X}$ vanishes for $q>n-\nu$.

The strategy of the proof of Theorem 0.2 is based on a direct application of the Bochner technique with special hermitian metrics constructed by means of the Calabi-Yau theorem.

Now, coming back to the principles of the proof of Theorem 0.1, we introduce the divisor

$$
D=\sum\left[\lambda_{j}\right] D_{j}
$$

Then Theorem 0.2 yields the vanishing of the map in cohomology

$$
H^{n-1}\left(X,-D+L+K_{X}\right) \longrightarrow H^{n-1}\left(X, L+K_{X}\right) .
$$

Thus we are reduced to show that $H^{n-1}\left(D, L+K_{X} \mid D\right)=0$, or dually that

$$
H^{0}(D,-L+D \mid D)=0
$$


This is now done by a detailed analysis of a potential nonzero section in $-L+D \mid D$; making use of the decomposition $(D)$ and of a Hodge index type inequality.

The vanishing Theorem 0.1 is most powerful when $X$ is a threefold, and in the second part of the paper we apply 0.1 - or rather a technical generalization - to prove the following abundance theorem:

Theorem 0.3. Let $X$ be a $\mathbb{Q}$-Gorenstein Kähler threefold with only terminal singularities, such that $K_{X}$ is nef ( a minimal Kähler threefold for short). Then $\kappa(X) \geq 0$.

This theorem was established in the projective case by Miyaoka and in [27] for Kähler threefolds, with the important exception that $X$ is a simple threefold which is not Kummer. Recall that $X$ is said to be simple if there is no proper compact subvariety through a very general point of $X$, and that $X$ is said to be Kummer if $X$ is bimeromorphic to a quotient of a torus. So our contribution here consists in showing that such a simple threefold $X$ with $K_{X}$ nef has actually $\kappa(X)=0$. Needless to say that among all Kähler threefolds the simple non-Kummer ones (which conjecturally do not exist) are most difficult to deal with, since they do not carry much global information besides the fact that $\pi_{1}$ is finite and that they have a holomorphic 2-form.

The first main ingredient in our approach is the inequality

$$
K_{X} \cdot c_{2}(X) \geq 0
$$

for a minimal simply connected Kähler threefold $X$ with algebraic dimension $a(X)=0$. Philosophically this inequality comes from Enoki's theorem that the tangent sheaf of $X$ is $K_{X}$-semi-stable when $K_{X}^{2} \neq 0$ resp. $\left(K_{X}, \omega\right)$-semi-stable when $K_{X}^{2}=0$; here $\omega$ is any Kähler form on $X$. Now if this semi-stability with respect to a degenerate polarization would yield a Miyaoka-Yau inequality, then $K_{X} \cdot c_{2}(X) \geq 0$ would follow. However this type of Miyoka-Yau inequalities with respect to degenerate polarizations is completey unknown. In the projective case, the inequality follows from Miyaoka's generic nefness theorem and is based on char. $p$-methods. Instead we approximate $K_{X}$ (in cohomology) by Kähler forms $\omega_{j}$. If $T_{X}$ is still $\omega_{j}$-semi-stable for sufficiently large $j$, then we can apply the usual Miyaoka-Yau inequality and pass to the limit to obtain $K_{X} \cdot c_{2}(X) \geq 0$. Otherwise we examine the maximal destabilizing subsheaf which essentially (because of $a(X)=0$ ) is independent of the polarization. 
The second main ingredient is the boundedness $h^{2}\left(X, m K_{X}\right) \leq 1$. If $K_{X}^{2} \neq 0$, this is of course contained in Theorem 0.1. If $K_{X}^{2}=0$, we prove this boundedness under the additional assumption that $a(X)=0$ and that $\pi_{1}(X)$ is finite (otherwise by a result of Campana $X$ is already Kummer). The main point is that if $h^{2}\left(X, m K_{X}\right) \geq 2$, then we obtain "many" non-split extensions

$$
0 \longrightarrow K_{X} \longrightarrow \mathcal{E} \longrightarrow m K_{X} \longrightarrow 0
$$

and we analyze whether $\mathcal{E}$ is semi-stable or not. The assumption on $\pi_{1}$ is used to conclude that if $\mathcal{E}$ is projectively flat, then $\mathcal{E}$ is trivial after a finite étale cover.

From these two ingredients Theorem 0.3 immediately follows by applying Riemann-Roch on a desingularization of $X$.

The only remaining problem concerning abundance on Kähler threefolds is to prove that a simple Kähler threefold with $K_{X}$ nef and $\kappa(X)=$ 0 must be Kummer.

\section{Preliminaries}

We start with a few preliminary definitions.

Definition 1.1. A normal complex space $X$ is said to be Kähler if there exists a Kähler form $\omega$ on the regular part of $X$ such that the following holds: Every singular point $x \in X$ admits an open neighborhood $U$ and a closed embedding $U \subset V$ into an open set $U \subset \mathbb{C}^{N}$ such that there is a Kähler form $\eta$ on $V$ with $\eta \mid U=\omega$.

Remark 1.2. Let $X$ be a compact Kähler space and let $f: \hat{X} \longrightarrow$ $X$ be a desingularization by a sequence of blow-ups. Then $\hat{X}$ is a Kähler manifold. More generally consider a holomorphic map $f: \hat{X} \longrightarrow X$ of a normal compact complex space to a normal compact Kähler space. If $f$ is a projective morphism or, more generally, a Kähler morphism, then $\hat{X}$ is Kähler. For references to this and more information on Kähler spaces, we refer to [31].

A Kähler form $\omega$ defines naturally a class $[\omega] \in H^{2}(X, \mathbb{R})$, see $[13]$ where Kähler metrics on singular spaces were first introduced. Therefore we also have a Kähler cone on a normal variety. 
Notation 1.3. Let $X$ be a normal compact complex space.

(1) Let $A$ and $B$ be reflexive sheaves of rank 1 . Then we define $A \hat{\otimes} B:=(A \otimes B)^{* *}$. Moreover we let $A^{[m]}:=A^{\hat{\otimes} m}$.

(2) A reflexive sheaf $A$ is said to be a $\mathbb{Q}$-line bundle if there exists a positive integer $m$ such that $A^{[m]}$ is locally free.

(3) $X$ is $\mathbb{Q}$-Gorenstein if the canonical reflexive sheaf $\omega_{X}$, also denoted $K_{X}$, is a $\mathbb{Q}$-line bundle. $X$ is $\mathbb{Q}$-factorial, if every reflexive sheaf of rank 1 is a $\mathbb{Q}$-line bundle.

Definition 1.4. Let $X$ be a normal compact Kähler threefold.

(1) $X$ is simple if there is no proper compact subvariety through the very general point of $X$.

(2) $X$ is Kummer, if $X$ is bimeromorphic to a quotient $T / G$ where $T$ is a torus and $G$ a finite group acting on $T$.

It is conjectured that all simple threefolds are Kummer.

\section{Notation 1.5.}

(1) The algebraic dimension $a(X)$ of an irreducible reduced compact complex space is the transcendence degree of the field of meromorphic functions over $\mathbb{C}$. If $a(X)=0$, i.e., all meromorphic functions on $X$ are constant, then it is well-known that $X$ carries only finitely many irreducible hypersurfaces.

(2) A line bundle $L$ on a compact Kähler manifold is nef, if $c_{1}(L)$ lies in the closure of the Kähler cone. For alternative descriptions see e.g., [8]. If $X$ is a normal compact Kähler, then $L$ is nef if there exists a desingularization $\pi: \hat{X} \longrightarrow X$ such that $\pi^{*}(L)$ is nef. By [26], this definition does not depend on the choice of $\pi$.

\section{Hodge index type inequalities}

We give here some generalizations of Hodge index inequalities for nef classes over compact Kähler manifolds. In this direction the main result is the Hovanskii-Teissier concavity inequality, which can be stated in the following way (see e.g., [7], Prop. 5.2 and Remark 5.3): 
Proposition 2.1. Let $\alpha_{1}, \ldots, \alpha_{k}$ and $\gamma_{1}, \ldots, \gamma_{n-k}$ be nef cohomology classes on a compact Kähler $n$-dimensional manifold $X$. Then

$$
\alpha_{1} \cdots \alpha_{k} \cdot \gamma_{1} \cdots \gamma_{n-k} \geqslant\left(\alpha_{1}^{k} \cdot \gamma_{1} \cdots \gamma_{n-k}\right)^{1 / k} \cdots\left(\alpha_{k}^{k} \cdot \gamma_{1} \cdots \gamma_{n-k}\right)^{1 / k} .
$$

We want to derive from these a non vanishing property for intersection products of the form $\alpha^{i} \cdot \beta^{j}$. Let us fix a Kähler metric $\omega$ on $X$. By Proposition 2.1 applied with $k=i+j$ and the $\alpha_{\ell}$ 's being $i$ copies of $\alpha$ followed by $j$ copies of $\beta$ and $\gamma_{\ell}=\omega$, we have

$$
\alpha^{i} \cdot \beta^{j} \cdot \omega^{n-i-j} \geqslant\left(\alpha^{k} \cdot \omega^{n-k}\right)^{i / k} \cdots\left(\beta^{k} \cdot \omega^{n-k}\right)^{j / k} .
$$

As all products $\alpha^{k}$ and analogues can be represented by closed positive currents, we have $\alpha^{k} \neq 0 \Rightarrow \alpha^{k} \cdot \omega^{n-k}>0$, hence with $k=i+j$ we find

$$
\alpha^{i+j} \neq 0 \text { and } \beta^{i+j} \neq 0 \Longrightarrow \alpha^{i} \cdot \beta^{j} \neq 0 \text {. }
$$

This is of course optimal in terms of the exponents if $\alpha=\beta$, but as we shall see in a moment, this is possibly not optimal in a dissymetric situation. Actually, we have the following additional inequalities which can be viewed as "differentiated" Hovanskii-Teissier inequalities:

Theorem 2.3. Let $\alpha$ and $\beta$ be nef cohomology classes of type $(1,1)$ on a compact Kähler $n$-dimensional manifold $X$. Assume that $\alpha^{p} \neq 0$ and $\beta^{q} \neq 0$ for some integers $p, q>0$. Then we have $\alpha^{i} \cdot \beta^{j} \neq 0$ as soon as there exists an integer $k \geqslant i+j$ such that

$$
i(k-p)_{+}+j(k-q)_{+}<k,
$$

where $x_{+}$means the positive part of a number $x$.

Proof. Assume that $\alpha^{i} \cdot \beta^{j}=0$. We apply the Hovanskii-Teissier inequality respectively with $\alpha_{\ell}=\alpha+\varepsilon \omega(i$ terms $)$, or $\alpha_{\ell}=\beta+\varepsilon \omega(j$ terms $)$ or $\alpha_{\ell}=\omega(k-i-j$ terms $)$, and $\gamma_{\ell}=\omega$. This gives

$$
\begin{aligned}
& (\alpha+\varepsilon \omega)^{i} \cdot(\beta+\varepsilon \omega)^{j} \cdot \omega^{n-i-j} \\
& \geqslant\left((\alpha+\varepsilon \omega)^{k} \cdot \omega^{n-k}\right)^{i / k}\left((\beta+\varepsilon)^{k} \cdot \omega^{n-k}\right)^{j / k}\left(\omega^{n}\right)^{1-i / k-j / k} .
\end{aligned}
$$

By expanding the intersection form and using the assumption $\alpha^{i} \cdot \beta^{j}=0$, we infer

$$
(\alpha+\varepsilon \omega)^{i} \cdot(\beta+\varepsilon \omega)^{j} \cdot \omega^{n-i-j} \leqslant O(\varepsilon)
$$


as $\varepsilon$ tends to zero. On the other hand $(\alpha+\varepsilon \omega)^{k} \cdot \omega^{n-k}$ is bounded away from 0 if $k \leqslant p$ since then $\alpha^{k} \neq 0$, and $(\alpha+\varepsilon \omega)^{k} \cdot \omega^{n-k} \geqslant C \varepsilon^{k-p}$ for some constant $C>0$ if $k \geqslant p$. Hence we infer from $(*)$ that

$$
C \varepsilon^{(i / k)(k-p)_{+}+(j / k)(k-q)_{+}}=O(\varepsilon),
$$

and this is not possible if $i(k-p)_{+}+j(k-q)_{+}<k$. The theorem is proved.

q.e.d.

The special case $p=2, q=1, i=j=1, k=2$ provides the following result which will be needed later on several occasions:

Corollary 2.4. Assume that $\alpha, \beta$ are nef with $\alpha^{2} \neq 0$ and $\beta \neq 0$. Then $\alpha \cdot \beta \neq 0$.

Finally, we state an extension of Proposition 2.1 in the case when one of the cohomology classes involved is not necessarily nef.

Proposition 2.5. Let $\alpha$ be a real $(1,1)$-cohomology class, and let $\beta, \gamma_{1}, \ldots \gamma_{n-2}$ be nef cohomology classes. Then

$$
\left(\alpha \cdot \beta \cdot \gamma_{1} \cdots \gamma_{n-2}\right)^{2} \geqslant\left(\alpha^{2} \cdot \gamma_{1} \cdots \gamma_{n-2}\right)\left(\beta^{2} \cdot \gamma_{1} \cdots \gamma_{n-2}\right) .
$$

Proof. By Proposition 2.1, the result is true when $\alpha$ is nef. If we replace $\beta$ by $\beta+\varepsilon \omega$ and let $\varepsilon>0$ tend to zero, we see that it is enough to consider the case when $\beta$ is a Kähler class. Then $\alpha+\lambda \beta$ is also Kähler for $\lambda \gg 1$ large enough, and the inequality holds true with $\alpha+\lambda \beta$ in place of $\alpha$. However, after making the replacement, the contributions of terms involving $\lambda$ in the right and left-hand side of the inequality are both equal to

$$
2 \lambda\left(\alpha \cdot \beta \cdot \gamma_{1} \cdots \gamma_{n-2}\right)\left(\beta^{2} \cdot \gamma_{1} \cdots \gamma_{n-2}\right)+\lambda^{2}\left(\beta^{2} \cdot \gamma_{1} \cdots \gamma_{n-2}\right)^{2} .
$$

Hence these terms cancel and the claim follows.

q.e.d.

\section{Partial vanishing for multiplier ideal sheaf cohomology}

Let $(L, h)$ be a holomorphic line bundle over a compact Kähler $n$ fold $X$. Locally in a trivialization $L_{\mid U} \simeq U \times \mathbb{C}$, the metric is given by $\|\xi\|_{x}=|\xi| e^{-\varphi(x)}$ and we assume that the curvature $\Theta_{h}(L):=\frac{i}{\pi} \partial \bar{\partial} \varphi$ is a closed positive current (so that, in particular, $L$ is pseudo-effective). We introduce as usual the multiplier ideal sheaf $\mathcal{I}(h):=\mathcal{I}(\varphi)$ where

$$
\mathcal{I}(\varphi)_{x}:=\left\{f \in \mathcal{O}_{X, x} ; \exists V \ni x, \quad \int_{V}|f(z)|^{2} e^{-2 \varphi(z)}<+\infty\right\}
$$


and $V$ is an arbitrarily small neighborhood of $x$. We also consider the upper regularized multiplier ideal sheaf

$$
\mathcal{I}_{+}(h):=\lim _{\varepsilon \rightarrow 0_{+}} \mathcal{I}\left(h^{1+\varepsilon}\right)=\lim _{\varepsilon \rightarrow 0_{+}} \mathcal{I}((1+\varepsilon) \varphi) .
$$

It should be noticed that $\mathcal{I}((1+\varepsilon) \varphi)$ increases as $\varepsilon$ decreases, hence the limit is locally stationary by the Noether property of coherent sheaves, and one has of course $\mathcal{I}_{+}(h) \subset \mathcal{I}(h)$. It is unknown whether these sheaves may actually differ (in all known examples they are equal). In any case, they coincide at least in codimension 1 (i.e., outside an analytic subset of codimension $\geqslant 2$ ).

Proposition 3.2. Let

$$
\Theta_{h}(L)=\sum_{j=1}^{+\infty} \lambda_{j} D_{j}+G
$$

be the Siu decomposition of the $(1,1)$-current $\Theta_{h}(L)$ as a countable sum of effective divisors and of a $(1,1)$-current $G$ such that the Lelong sublevel sets $E_{c}(G), c>0$, all have codimension 2 . Then we have the inclusion of sheaves

$$
\mathcal{I}_{+}(h) \subset \mathcal{I}(h) \subset \mathcal{O}\left(-\sum\left[\lambda_{j}\right] D_{j}\right), \quad\left[\lambda_{j}\right]:=\text { integer part of } \lambda_{j},
$$

and equality holds on $X \backslash Z$ where $Z$ is an analytic subset of $X$ whose components all have codimension at least 2.

Proof. The decomposition exists by [29] (see also [6]). Now, if $g_{j}$ is a local generator of the ideal sheaf $\mathcal{O}\left(-D_{j}\right)$, the plurisubharmonic weight $\varphi$ of $h$ can be written as

$$
\varphi=\sum \lambda_{j} \log \left|g_{j}\right|+\psi
$$

where $\psi$ is plurisubharmonic and the $E_{c}(\psi)$ have codimension 2 at least. Since $\psi$ is locally bounded from above, it is obvious that

$$
\mathcal{I}(\varphi) \subset \mathcal{I}\left(\lambda_{j} \log \left|g_{j}\right|\right) \subset \mathcal{O}\left(-\sum\left[\lambda_{j}\right] D_{j}\right) .
$$

Now, let $Y$ be the union of all sets $E_{c}(\psi)$ (with, say, $c=1 / k$ ), all pairwise intersections $D_{j} \cap D_{k}$ and all singular sets $D_{j \text { sing. This set }}$ $Y$ is at most a countable union of analytic sets of codimension $\geqslant 2$. Pick an arbitrary point $x \in X \backslash Y$. Then $x$ meets the support of $\bigcup D_{j}$ 
in at most one point which is then a smooth point of some $D_{k}$, and the Lelong number of $\psi_{k}=\psi+\sum_{j \neq k} \lambda_{j} \log \left|g_{j}\right|$ at $x$ is zero. Then $\varphi=\lambda_{k} \log \left|g_{k}\right|+\psi_{k}$ and the inclusion

$$
\mathcal{I}(h)_{x} \supset \mathcal{O}\left(-\sum_{j}\left[\lambda_{j}\right] D_{j}\right)_{x}=\mathcal{O}\left(-\left[\lambda_{k}\right] D_{k}\right)_{x}
$$

holds true by Hölder's inequality. In fact, for every germ $f$ in $\mathcal{O}\left(-\left[\lambda_{k}\right] D_{k}\right)_{x}$ we have

$$
\int_{V \ni x}|f|^{2} \exp \left(-(1+\varepsilon) \lambda_{k} \log \left|g_{k}\right|\right)<+\infty
$$

for $\varepsilon>0$ so small that $\left[(1+\varepsilon) \lambda_{k}\right]=\left[\lambda_{k}\right]$, while $e^{-\psi_{k}}$ is in $L^{p}\left(V_{p}\right)$ for some $V_{p} \ni x$, for every $p>1$. Similarly, we have

$$
\mathcal{I}_{+}(h)_{x} \supset \mathcal{O}\left(-\left[(1+\varepsilon) \lambda_{k}\right] D_{k}\right)_{x}=\mathcal{O}\left(-\left[\lambda_{k}\right] D_{k}\right)_{x}
$$

for $\varepsilon>0$ small enough. The analytic set $Z$ where our sheaves differ [i.e., the union of supports of $\mathcal{I}(h) / \mathcal{I}_{+}(h)$ and $\left.\mathcal{O}\left(-\sum_{j}\left[\lambda_{j}\right] D_{j}\right) / \mathcal{I}(h)\right]$ must be contained in $Y$, hence $Z$ is of codimension $\geqslant 2$.

The main goal of this section is to prove the following partial vanishing theorem:

Theorem 3.3. Let $(L, h)$ be a holomorphic line bundle over a compact Kähler $n$-fold $X$, equipped with a singular metric $h$ such that $\Theta_{h}(L) \geqslant 0$. Assume that $L$ is nef and has numerical dimension $\nu(L)=$ $\nu \geqslant 0$, i.e., $c_{1}(L)^{\nu} \neq 0$ and $\nu$ is maximal. Then the morphism

$$
H^{q}\left(X, \mathcal{O}\left(K_{X}+L\right) \otimes \mathcal{I}_{+}(h)\right) \longrightarrow H^{q}\left(X, K_{X}+L\right)
$$

induced by the inclusion $\mathcal{I}_{+}(h) \subset \mathcal{O}_{X}$ vanishes for $q>n-\nu$.

Of course, it is expected that the Kawamata-Viehweg vanishing theorem also holds for Kähler manifolds, in which case the whole group $H^{q}\left(X, K_{X}+L\right)$ vanishes and Theorem 3.3 would then be an obvious consequence. However, we will see in Section 4 that, conversely, Theorem 3.3 can be used to derive the Kawamata-Viehweg vanishing theorem in the first nontrivial case $\nu=2$. Using the same method for higher values of $\nu$ would probably be very hard, if not impossible.

Proof. Our strategy is based on a direct application of the Bochner technique with special hermitian metrics constructed by means of the Calabi-Yau theorem. 
Let us fix a smooth hermitian metric $h_{\infty}$ on $L$, which may have a curvature form $\Theta_{h_{\infty}}(L)$ of arbitrary sign, and let $\varepsilon>0$. Then $c_{1}(L)+\varepsilon \omega$ is a Kähler class, hence by the Calabi-Yau theorem for complex MongeAmpère equations there exists a hermitian metric $h_{\varepsilon}=h_{\infty} e^{-2 \varphi_{\varepsilon}}$ such that

$$
\left(\Theta_{h_{\varepsilon}}(L)+\varepsilon \omega\right)^{n}=C_{\varepsilon} \omega^{n} .
$$

Here $C_{\varepsilon}>0$ is the constant such that

$$
C_{\varepsilon}=\frac{\int_{X}\left(c_{1}(L)+\varepsilon \omega\right)^{n}}{\int_{X} \omega^{n}} \geqslant C \varepsilon^{n-\nu} .
$$

Let $h=h_{\infty} e^{-2 \psi}$ be a metric with $\Theta_{h}(L) \geqslant 0$ as given in the statement of the theorem, and let $\psi_{\varepsilon} \downarrow \psi$ be a regularization of $\psi$ possessing only analytic singularities (i.e., only logarithmic poles), such that

$$
\widetilde{h}_{\varepsilon}:=h_{\infty} e^{-2 \psi_{\varepsilon}}
$$

satisfies $\Theta_{\widetilde{h}_{\varepsilon}}(L) \geqslant-\varepsilon \omega$ in the sense of currents. Such a metric exists by the general regularization results proved in [5]. We consider the metric

$$
\hat{h}_{\varepsilon}=\left(h_{\varepsilon}\right)^{\delta}\left(\widetilde{h}_{\varepsilon}\right)^{1-\delta}=h_{\infty} \exp \left(-2\left(\delta \varphi_{\varepsilon}+(1-\delta) \psi_{\varepsilon}\right)\right)
$$

where $\delta>0$ is a sufficiently small number which will be fixed later. By construction,

$$
\begin{aligned}
\Theta_{\hat{h}_{\varepsilon}}(L)+2 \varepsilon \omega & =\delta\left(\Theta_{h_{\varepsilon}}(L)+\varepsilon \omega\right)+(1-\delta)\left(\Theta_{\widetilde{h}_{\varepsilon}}(L)+\varepsilon \omega\right)+\varepsilon \omega \\
& \geqslant \delta\left(\Theta_{h_{\varepsilon}}(L)+\varepsilon \omega\right)+\varepsilon \omega .
\end{aligned}
$$

Denote by $\lambda_{1} \leqslant \cdots \leqslant \lambda_{n}$ and $\hat{\lambda}_{1} \leqslant \cdots \leqslant \hat{\lambda}_{n}$, respectively, the eigenvalues of the curvature forms $\Theta_{h_{\varepsilon}}(L)+\varepsilon \omega$ and $\Theta_{\hat{h}_{\varepsilon}}(L)+2 \varepsilon \omega$ at every point $z \in X$, with respect to the base Kähler metric $\omega(z)$. By the minimax principle we find $\hat{\lambda}_{j} \geqslant \delta \lambda_{j}+\varepsilon$. On the other hand, the Monge-Ampère equation (3.4) tells us that

$$
\lambda_{1} \ldots \lambda_{n}=C_{\varepsilon} \geqslant C \varepsilon^{n-\nu}
$$

everywhere on $X$. We apply the basic Bochner-Kodaira inequality to sections of type $(n, q)$ with values in the hermitian line bundle $\left(L, \hat{h}_{\varepsilon}\right)$. As the curvature eigenvalues of $\Theta_{\hat{h}_{\varepsilon}}(L)$ are equal to $\hat{\lambda}_{j}-2 \varepsilon$ by definition, we find

$$
\|\bar{\partial} u\|_{\hat{h}_{\varepsilon}}^{2}+\left\|\bar{\partial}^{\star} u\right\|_{\hat{h}_{\varepsilon}}^{2} \geqslant \int_{X}\left(\hat{\lambda}_{1}+\cdots+\hat{\lambda}_{q}-2 q \varepsilon\right)|u|_{\hat{h}_{\varepsilon}}^{2} d V_{\omega}
$$


for every smooth $(n, q)$-form $u$ with values in $L$. Actually this is formally true only if the metric $\hat{h}_{\varepsilon}$ is smooth on $X$. The metric $h_{\varepsilon}$ is indeed smooth, but $\widetilde{h}_{\varepsilon}$ may have poles along an analytic set $Z_{\varepsilon} \subset X$. In that case, we apply instead the inequality to forms $u$ which are compactly supported in $X \backslash Z_{\varepsilon}$, and replace the Kähler metric $\omega$ by a sequence of complete Kähler metrics $\omega_{k} \downarrow \omega$ on $X \backslash Z_{\varepsilon}$, and pass to the limit as $k$ tends to $+\infty$ (see e.g., [5] for details about such techniques). In the limit we recover the same estimates as if we were in the smooth case, and we therefore allow ourselves to ignore this minor technical problem from now on.

Now, let us take a cohomology class $\{\beta\} \in H^{q}\left(X, K_{X} \otimes L \otimes \mathcal{I}_{+}(h)\right)$. By using Cech cohomology and the De Rham-Weil isomorphism between Cech and Dolbeault cohomology (via a partition of unity and the usual homotopy formulas), we obtain a representative $\beta$ of the cohomology class which is a smooth $(n, q)$-form with values in $L$, such that the coefficients of $\beta$ lie in the sheaf $\mathcal{I}_{+}(h) \otimes_{\mathcal{O}_{X}} \mathcal{C}^{\infty}$. We want to show that $\beta$ is a boundary with respect to the cohomology group $H^{q}\left(X, K_{X} \otimes\right.$ $L)$. This group is a finite dimensional Hausdorff vector space whose topology is induced by the $L^{2}$ Hilbert space topology on the space of forms (all Sobolev norms induce in fact the same topology on the level of cohomology groups). Therefore, it is enough to show that we can approach $\beta$ by $\bar{\partial}$-exact forms in $L^{2}$ norm.

As in Hörmander [15], we write every form $u$ in the domain of the $L^{2}$-extension of $\bar{\partial}^{*}$ as $u=u_{1}+u_{2}$ with

$$
u_{1} \in \operatorname{Ker} \bar{\partial} \quad \text { and } \quad u_{2} \in(\operatorname{Ker} \bar{\partial})^{\perp}=\overline{\operatorname{Im} \bar{\partial}^{*}} \subset \operatorname{Ker} \bar{\partial}^{*} .
$$

Therefore, since $\beta \in \operatorname{Ker} \bar{\partial}$,

$$
\begin{aligned}
\left.|\langle\beta, u\rangle\rangle\right|^{2} & =\left|\left\langle\left\langle\beta, u_{1}\right\rangle\right\rangle\right|^{2} \\
& \leqslant \int_{X} \frac{1}{\hat{\lambda}_{1}+\cdots+\hat{\lambda}_{q}}|\beta|_{\hat{h}_{\varepsilon}}^{2} d V_{\omega} \int_{X}\left(\hat{\lambda}_{1}+\cdots+\hat{\lambda}_{q}\right)\left|u_{1}\right|_{\hat{h}_{\varepsilon}}^{2} d V_{\omega} .
\end{aligned}
$$

As $\bar{\partial} u_{1}=0$, an application of (3.6) to $u_{1}$ (together with an approximation of $u_{1}$ by compactly supported smooth sections on the corresponding complete Kähler manifold $X \backslash Z_{\varepsilon}$ ) shows that the second integral in the right-hand side is bounded above by

$$
\left\|\bar{\partial}^{*} u_{1}\right\|_{\hat{h}_{\varepsilon}}^{2}+2 q \varepsilon\left\|u_{1}\right\|_{\hat{h}_{\varepsilon}}^{2} \leqslant\left\|\bar{\partial}^{*} u\right\|_{\hat{h}_{\varepsilon}}^{2}+2 q \varepsilon\|u\|_{\hat{h}_{\varepsilon}}^{2}
$$

so we finally get

$$
\left.|\langle\beta, u\rangle\rangle\right|^{2} \leqslant \int_{X} \frac{1}{\hat{\lambda}_{1}+\cdots+\hat{\lambda}_{q}}|\beta|_{\hat{h}_{\varepsilon}}^{2} d V_{\omega}\left(\left\|\bar{\partial}^{*} u\right\|_{\hat{h}_{\varepsilon}}^{2}+2 q \varepsilon\|u\|_{\hat{h}_{\varepsilon}}^{2}\right) .
$$


By the Hahn-Banach theorem (or rather a Hilbert duality argument in this situation), we can find elements $v_{\varepsilon}, w_{\varepsilon}$ such that

$$
\langle\langle\beta, u\rangle\rangle=\left\langle\left\langle v_{\varepsilon}, \bar{\partial}^{*} u\right\rangle\right\rangle+\left\langle\left\langle w_{\varepsilon}, u\right\rangle\right\rangle \quad \forall u, \quad \text { i.e., } \quad \beta=\bar{\partial} v_{\varepsilon}+w_{\varepsilon},
$$

with

$$
\left\|v_{\varepsilon}\right\|_{\hat{h}_{\varepsilon}}^{2}+\frac{1}{2 q \varepsilon}\left\|w_{\varepsilon}\right\|_{\hat{h}_{\varepsilon}}^{2} \leqslant \int_{X} \frac{1}{\hat{\lambda}_{1}+\cdots+\hat{\lambda}_{q}}|\beta|_{\hat{h}_{\varepsilon}}^{2} d V_{\omega} .
$$

As a consequence, the $L^{2}$ distance of $\beta$ to the space of $\bar{\partial}$-exact forms is bounded by $\left\|w_{\varepsilon}\right\|_{\hat{h}_{\varepsilon}}$ where

$$
\begin{aligned}
\left\|w_{\varepsilon}\right\|_{\hat{h}_{\varepsilon}}^{2} & =\int_{X}\left|w_{\varepsilon}\right|_{h_{\infty}}^{2} e^{-2\left(\delta \varphi_{\varepsilon}+(1-\delta) \psi_{\varepsilon}\right)} d V_{\omega} \\
& \leqslant 2 q \varepsilon \int_{X} \frac{1}{\hat{\lambda}_{1}+\cdots+\hat{\lambda}_{q}}|\beta|_{\hat{h}_{\varepsilon}}^{2} d V_{\omega}
\end{aligned}
$$

We normalize the choice of the potentials $\varphi_{\varepsilon}, \psi$ and $\psi_{\varepsilon}$ so that

$$
\sup _{X} \varphi_{\varepsilon}=0, \quad \sup _{X} \psi=-1, \quad-1 \leqslant \sup _{X} \psi_{\varepsilon}<0
$$

in this way $\varphi_{\varepsilon}, \psi_{\varepsilon} \leqslant 0$ everywhere on $X$ (all inequalities can be achieved simply by adding suitable constants). From this we infer

$$
\int_{X}\left|w_{\varepsilon}\right|_{h_{\infty}}^{2} d V_{\omega} \leqslant 2 \int_{X} \frac{q \varepsilon}{\hat{\lambda}_{1}+\cdots+\hat{\lambda}_{q}}|\beta|_{\hat{h}_{\varepsilon}}^{2} d V_{\omega}
$$

and what remains to be shown is that the right-hand side converges to 0 for a suitable choice of $\delta>0$. By construction $\hat{\lambda}_{j} \geqslant \delta \lambda_{j}+\varepsilon$ and (3.5) implies

$$
\lambda_{q}^{q} \lambda_{q+1} \ldots \lambda_{n} \geqslant \lambda_{1} \ldots \lambda_{n} \geqslant C \varepsilon^{n-\nu}
$$

hence

$$
\frac{1}{\lambda_{1}+\cdots+\lambda_{q}} \leqslant \frac{1}{\lambda_{q}} \leqslant C^{-1 / q} \varepsilon^{-(n-\nu) / q}\left(\lambda_{q+1} \ldots \lambda_{n}\right)^{1 / q} .
$$

We infer

$$
\begin{aligned}
\gamma_{\varepsilon}: & =\frac{q \varepsilon}{\hat{\lambda}_{1}+\cdots+\hat{\lambda}_{q}} \leqslant \min \left(1, \frac{q \varepsilon}{\delta \lambda_{q}}\right) \\
& \leqslant \min \left(1, C \delta^{-1} \varepsilon^{1-(n-\nu) / q}\left(\lambda_{q+1} \ldots \lambda_{n}\right)^{1 / q}\right) .
\end{aligned}
$$


We notice that

$$
\begin{aligned}
\int_{X} \lambda_{q+1} \ldots \lambda_{n} d V_{\omega} & \leqslant \int_{X}\left(\Theta_{h_{\varepsilon}}(L)+\varepsilon \omega\right)^{n-q} \wedge \omega^{q} \\
& =\left(c_{1}(L)+\varepsilon\{\omega\}\right)^{n-q}\{\omega\}^{q} \leqslant C^{\prime \prime},
\end{aligned}
$$

hence the functions $\left(\lambda_{q+1} \ldots \lambda_{n}\right)^{1 / q}$ are uniformly bounded in $L^{1}$ norm as $\varepsilon$ tends to zero. Since $1-(n-\nu) / q>0$ by hypothesis, we conclude that $\gamma_{\varepsilon}$ converges almost everywhere to 0 as $\varepsilon$ tends to zero. On the other hand

$$
|\beta|_{\hat{h}_{\varepsilon}}^{2}=|\beta|_{h_{\infty}}^{2} e^{-2\left(\delta \varphi_{\varepsilon}+(1-\delta) \psi_{\varepsilon}\right)} \leqslant|\beta|_{h_{\infty}}^{2} e^{-2 \delta \varphi_{\varepsilon}} e^{-2 \psi} .
$$

Our assumption that the coefficients of $\beta$ lie in $\mathcal{I}_{+}(h)$ implies that there exists $p^{\prime}>1$ such that $\int_{X}|\beta|_{h_{\infty}}^{2} e^{-2 p^{\prime} \psi} d V_{\omega}<\infty$. Let $\left.p \in\right] 1,+\infty[$ be the conjugate exponent such that $\frac{1}{p}+\frac{1}{p^{\prime}}=1$. By Hölder's inequality, we have

$$
\begin{aligned}
& \int_{X} \gamma_{\varepsilon}|\beta|_{\hat{h}_{\varepsilon}}^{2} d V_{\omega} \\
& \leqslant\left(\int_{X}|\beta|_{h_{\infty}}^{2} e^{-2 p \delta \varphi_{\varepsilon}} d V_{\omega}\right)^{1 / p}\left(\int_{X} \gamma_{\varepsilon}^{p^{\prime}}|\beta|_{h_{\infty}}^{2} e^{-2 p^{\prime} \psi} d V_{\omega}\right)^{1 / p^{\prime}} .
\end{aligned}
$$

As $\gamma_{\varepsilon} \leqslant 1$, the Lebesgue dominated convergence theorem shows that

$$
\int_{X} \gamma_{\varepsilon}^{p^{\prime}}|\beta|_{h_{\infty}}^{2} e^{-2 p^{\prime} \psi} d V_{\omega}
$$

converges to 0 as $\varepsilon$ tends to 0 . However, the family of quasi plurisubharmonic functions $\left(\varphi_{\varepsilon}\right)$ is a bounded family with respect to the $L^{1}$ topology on the space of (quasi)-plurisubharmonic functions - we use here the fact that the currents

$$
\Theta_{\widetilde{h}_{\varepsilon}}(L)=\Theta_{h_{\infty}}(L)+\frac{i}{\pi} \partial \bar{\partial} \varphi_{\varepsilon} \geqslant 0
$$

all sit in the same cohomology class; the boundedness of their normalized potentials then results from the continuity properties of the Green operator. By standard results of complex potential theory, we conclude that there exists a small constant $\eta>0$ such that $\int_{X} e^{-2 \eta \varphi_{\varepsilon}} d V_{\omega}$ is uniformly bounded; in fact this follows from Prop. 7.1 of [30], p. 389, and from the arguments of its proof. By choosing $\delta \leqslant \eta / p$, the integral $\int_{X}|\beta|_{h_{\infty}}^{2} e^{-2 p \delta \varphi_{\varepsilon}} d V_{\varepsilon}$ remains bounded and we are done. $\quad$ q.e.d. 


\section{Kawamata-Viehweg vanishing theorem for line bundles of numerical dimension 2}

In this section we prove the Kawamata-Viehweg vanishing theorem for the cohomology group of degree $n-1$ of nef line bundles $L$ with $L^{2} \neq 0$ on compact Kähler spaces of dimension $n$. Furthermore we will prove an extended version where $L$ can be a reflexive sheaf. This will be needed for proving the abundance theorem for Kähler threefolds.

Theorem 4.1. Let $X$ be a normal compact Kähler space of dimension $n$ and $L$ a nef line bundle on $X$. Assume that $L^{2} \neq 0$. Then

$$
H^{q}\left(X, K_{X}+L\right)=0
$$

for $q \geq n-1$.

Proof. In a first step we reduce the proof to the case of a smooth space $X$ (this is comfortable but not really necessary; all arguments would also work in the singular setting as well). In fact, let $\pi: \hat{X} \longrightarrow X$ be a Kähler desingularization. Then, assuming our claim in the smooth case, we have

$$
H^{q}\left(\hat{X}, K_{\hat{X}}+\pi^{*}(L)\right)=0 .
$$

By the projection formula and the Grauert-Riemenschneider vanishing theorem

$$
R^{j} \pi_{*}\left(K_{\hat{X}}\right)=0
$$

it follows

$$
H^{q}\left(X, \pi_{*}\left(K_{\hat{X}}\right) \otimes L\right)=0 .
$$

Since $\pi_{*}\left(K_{\hat{X}}\right) \subset K_{X}$ with cokernel supported in codimension at least 2, namely on the singular locus of $X$, the vanishing claim follows.

So from now on, we assume $X$ smooth. In the case $q=n$, we have $H^{n}\left(X, K_{X}+L\right)=H^{0}(X,-L)^{*}$ by Serre duality, and for $L$ nef, $-L$ has no section unless $L$ is trivial. Therefore the only interesting case is $q=n-1$. We introduce a singular metric $h$ on $L$ with positive curvature current $T$. By $[29]$ and $[5,6]$ we obtain a decomposition

$$
T=\sum \lambda_{j} D_{j}+G
$$

where $\lambda_{j} \geq 1$ are irreducible divisors, and $G$ is a positive current such that $G$ has Lelong numbers in codimension $\geqslant 2$ only - so that in 
particular $G \mid D_{i}$ is pseudo-effective for all $i$. Consider the multiplier ideal sheaf $\mathcal{I}(h)$. By Proposition 3.2 we have

$$
\mathcal{I}_{+}(h) \subset \mathcal{I}(h) \subset \mathcal{O}_{X}\left(-\sum\left[\lambda_{j}\right] D_{j}\right)
$$

with equality in codimension 1 . We put

$$
D=\sum\left[\lambda_{j}\right] D_{j}
$$

We consider the canonical map in cohomology

$$
H^{n-1}\left(X,-D+L+K_{X}\right) \longrightarrow H^{n-1}\left(X, L+K_{X}\right)
$$

which is vanishing by (3.3). In order to prove our claim it is therefore sufficient to prove

$$
H^{n-1}\left(D, L+K_{X} \mid D\right)=0 .
$$

By Serre duality and the adjunction formula, this comes down to showing

$$
H^{0}(D,-L+D \mid D)=0 .
$$

Supposing the contrary, we fix a nonzero section

$$
\sigma \in H^{0}(D,-L+D) \text {. }
$$

We choose $p_{1}, \ldots, p_{k}$ maximal so that

$$
\sigma \in H^{0}\left(D,-L+D-\sum p_{j} D_{j}\right)
$$

i.e., we choose $\widetilde{D}=\sum p_{j} D_{j} \subset D$ maximal such that $\sigma \mid \widetilde{D}=0$. $[$ In this notation, we view $\widetilde{D}$ as the subscheme of $X$ defined by the structure sheaf $\left.\mathcal{O}_{X} / \mathcal{O}_{X}(-\widetilde{D})\right]$

Then $0 \leq p_{i} \leq\left[\lambda_{i}\right]$ for all $i \in I$, not all $p_{i}=\left[\lambda_{i}\right]$, and we shall always consider $\sigma$ as a section of $-L+D-\sum p_{j} D_{j}$. Denote

$$
c_{i}=\frac{\left\{\lambda_{i}\right\}+p_{i}}{\lambda_{i}} .
$$

Then we have $0 \leq c_{i} \leq 1$. We introduce $c=\min c_{i}$ and

$$
I_{0}=\left\{i \in I \mid c_{i}=c\right\} .
$$

Clearly $c<1$. Notice that by construction $\sigma \mid D_{i} \neq 0$ unless $c_{i}=1$. Let

$$
E=-\left(\sum\left(\left\{\lambda_{i}\right\}+p_{i}\right) D_{i}\right)-G .
$$


Since $L=\sum \lambda_{i} D_{i}+G$, we have

$$
-L+D-\sum p_{i} D_{i}=-\left(\sum\left(\left\{\lambda_{i}\right\}+p_{i}\right) D_{i}\right)-G=E,
$$

so $E$ is effective (possibly zero) on every $D_{i}$ with $c_{i}<1$. Since $L$ is nef, also the $\mathbb{R}$-divisor $c L=\sum \lambda_{i} c D_{i}+c G$ is nef. Adding this to the divisor $E$ in the last equation, we deduce that

$$
-\left(\sum\left(\left\{\lambda_{i}\right\}+p_{i}-c \lambda_{i}\right) D_{i}\right)-(1-c) G
$$

is pseudo-effective on every $D_{i}$ with $c_{i}<1$. Since $\left\{\lambda_{i}\right\}+p_{i}-c \lambda_{i}=0$ for $i \in I_{0}$, it follows that

$$
-\left(\sum_{i \notin I_{0}}\left(\left\{\lambda_{i}\right\}+p_{i}-c \lambda_{i}\right) D_{i}\right)-(1-c) G
$$

is pseudo-effective on every $D_{i}$ with $c_{i}<1$, in particular for every $i \in I_{0}$. Now $D_{j} \mid D_{i}$ is effective (possibly 0 ) for all $j \neq i$, and $G \mid D_{i}$ is always pseudo-effective, hence, having in mind $c<1$ and $\left\{\lambda_{i}\right\}+p_{i}-c \lambda_{i}>0$ for $i \notin I_{0}$, we conclude that

$$
D_{j} \mid D_{i} \equiv 0
$$

for all $(j, i)$ with $j \notin I_{0}$ and $i \in I_{0}$ and that

$$
G \mid D_{i} \equiv 0
$$

for all $i \in I_{0}$. Introducing

$$
D^{\prime}=\sum_{i \in I_{0}} \lambda_{i} D_{i}
$$

and

$$
D^{\prime \prime}=\sum_{i \notin I_{0}} \lambda_{i} D_{i}
$$

we have

$$
L=D^{\prime}+D^{\prime \prime}+G
$$

and $D^{\prime \prime} \cdot D_{i}=G \cdot D_{i}=0$ for all $i \in I_{0}$ by (1) and (2). Hence $L \cdot D_{i}=D^{\prime} \cdot D_{i}$ for $i \in I_{0}$, so that $D^{\prime} \mid D_{i}$ is nef, hence $D^{\prime}$ is nef by [26]. In total

$$
L \cdot D^{\prime}=D^{\prime} \cdot D^{\prime}
$$


and

$$
D^{\prime} \cdot D^{\prime \prime}=D^{\prime} \cdot G=0 .
$$

As $L^{2} \neq 0$ and $D^{\prime} \neq 0$, Corollary 2.4 implies $L \cdot D^{\prime} \neq 0$. First recall that

$$
L\left|D_{i}=\sum_{j \in I_{0}} \lambda_{j} D_{j}\right| D_{i}
$$

is nef for $i \in I_{0}$. On the other hand

$$
\left.-\sum_{j \in I_{0}}\left\{\lambda_{j}\right\}+p_{j}\right) D_{j}=-c \sum_{j \in I_{0}} \lambda_{j} D_{j}
$$

is of course pseudo-effective on every $D_{i}$ for $i \in I_{0}$ ( $E$ is effective on those $D_{i}$ ). Combining these two facts, we deduce that either $c=0$ or that $L \cdot D_{i}=0$ for all $i \in I_{0}$, hence $L \cdot D^{\prime}=0$, contradiction. So we have $c=0$. This means $p_{j}=0$ and $\lambda_{j} \in \mathbb{N}$ for all $j \in I_{0}$.

Claim 4.2. The divisor $D^{\prime \prime}+G$ is nef, and in fact must be equal to zero.

Proof of the claim. We consider the closed positive $(1,1)$-current $\Theta=\left[D^{\prime \prime}\right]+G$. By the results of $[26]$, the proof of nefness of $\{\Theta\}$ just amounts to showing that the restriction $\{\Theta\}_{\mid Z}$ of the $(1,1)$-cohomology class $\{\Theta\}$ to any component $Z$ in the Lelong sublevel set $\bigcup_{c>0} E_{c}(\Theta)$ is nef. However $Z$ is either a component of $D^{\prime \prime}$ or a component of $\bigcup_{c>0} E_{c}(G)$. In the first case, $Z$ is contained in the support of $D^{\prime \prime}$, and as $D^{\prime} \cdot D^{\prime \prime}=0, Z$ must be disjoint from $D^{\prime}$. Hence

$$
\{\Theta\}_{\mid Z}=\left\{D^{\prime \prime}+G\right\}_{\mid Z}=\left\{L-D^{\prime}\right\}_{\mid Z}=\{L\}_{\mid Z}
$$

is nef. If $Z$ is a component of $\bigcup_{c>0} E_{c}(G)$, then $Z$ has codimension at least 2 . Then we know by [6] that the intersection product $\left[D^{\prime}\right] \wedge G$ is well-defined as a closed positive current. Since the cohomology class of this current is zero, we must have $\left[D^{\prime}\right] \wedge G=0$. However, we infer from [6] that

$$
\nu\left(\left[D^{\prime}\right] \wedge G, z\right) \geqslant \nu\left(\left[D^{\prime}\right], z\right) \nu(G, z)>0
$$

at every point $z \in D^{\prime} \cap Z$, hence $Z$ must also be disjoint from $D^{\prime}$ in that case. We conclude as before that $\{\Theta\}_{\mid Z}=\{L\}_{\mid Z}$ is nef. Now we have $D^{\prime} \cdot\left(D^{\prime \prime}+G\right)=0$, with $D^{\prime}, D^{\prime \prime}+G$ nef and $D^{\prime 2}=L \cdot D^{\prime} \neq 0$. Hence $\left\{D^{\prime \prime}+G\right\}=0$ by Corollary 2.4 , and we conclude that $D^{\prime \prime}=0, G=0$ (both $\left[D^{\prime \prime}\right]$ and $[G]$ being positive currents). $\quad$ q.e.d.

From this we infer $L \equiv D^{\prime}$ and $\mathcal{I}(h)=\mathcal{O}_{X}\left(-D^{\prime}\right)$. 
Case 1. We assume that $L=D^{\prime}$. Now the sequence

$$
0 \longrightarrow \mathcal{I}(h) \otimes K_{X}+L \longrightarrow K_{X}+L \longrightarrow K_{X}+L \mid D^{\prime}=K_{D^{\prime}} \longrightarrow 0
$$

gives in cohomology

$$
\begin{aligned}
0 \longrightarrow H^{n-1}\left(X, K_{X}+L\right) & \longrightarrow H^{n-1}\left(D^{\prime}, K_{D^{\prime}}\right) \\
& \simeq H^{0}\left(D^{\prime}, \mathcal{O}_{D^{\prime}}\right) \longrightarrow H^{n}\left(K_{X}\right)=\mathbb{C} \longrightarrow 0
\end{aligned}
$$

Thus we need to show

$$
h^{0}\left(D^{\prime}, \mathcal{O}_{D^{\prime}}\right)=1 .
$$

In order to verify this, we first observe that $D^{\prime}$ is connected. In fact otherwise write $D^{\prime}=A+B$ with $A$ and $B$ effective and $A \cdot B=0$. But $A$ and $B$ are necessarily nef, hence the Hodge Index Theorem gives a contradiction to $L^{2}=\left(D^{\prime}\right)^{2} \neq 0$. So $D^{\prime}$ is connected and if $h^{0}\left(D^{\prime}, \mathcal{O}_{D^{\prime}}\right) \geq 2$, then $\mathcal{O}_{D^{\prime}}$ contains a nilpotent section $t \neq 0$. Let $\sum_{j \in I} \mu_{j} D_{j}$ denote its vanishing divisor (notice that $D^{\prime}$ is Cohen-Macaulay!). Then $1 \leq \mu_{j} \leq \lambda_{j}$ for all $j$. Let

$$
J=\left\{j \in J \mid \frac{\lambda_{j}}{\mu_{j}} \text { maximal }\right\}
$$

and let $c=\frac{\lambda_{j}}{\mu_{j}}$ be the maximal value. Notice that $-\sum_{j \in I} \mu_{j} D_{j} \mid D_{i}$ is effective (possibly 0) for all $i$. First we rule out the case that $c=\frac{\lambda_{j}}{\mu_{j}}$ for all $j \in I$. In fact, then $L\left|D_{i}=c \sum \mu_{j} D_{j}\right| D_{i}$ is nef and its dual is effective, hence $L \mid D_{i} \equiv 0$ for all $i$, whence $L^{2}=0$, contradiction. Thus we find some $j$ such that

$$
c>\frac{\lambda_{j}}{\mu_{j}} .
$$

By connectedness of $D=D^{\prime}$ we can choose $i_{0} \in J$ in such a way that there exists $j_{1} \in I \backslash J$ with $D_{i_{0}} \cap D_{j_{1}} \neq \emptyset$. Now

$$
\sum_{j \in I}\left(\lambda_{j}-c \mu_{j}\right) D_{j} \mid D_{i_{0}}
$$

is pseudo-effective as a sum of a nef and an effective line bundle (this has nothing to do with the choice of $i_{0}$ ). Since the sum, taken over $I$, is the same as the sum taken over $I \backslash\left\{i_{0}\right\}$, we conclude that

$$
\sum_{j \neq i_{0}}\left(\lambda_{j}-\mu_{j}\right) D_{j} \mid D_{i_{0}}
$$


is pseudo-effective, too. Now all $\lambda_{j}-c \mu_{j} \leq 0$ and $\lambda_{j_{1}}-c \mu_{j_{1}}<0$ with $D_{j_{1}} \cap D_{i_{0}} \neq \emptyset$, hence the dual of

$$
\sum_{j \neq i_{0}}\left(\lambda_{j}-\mu_{j}\right) D_{j} \mid D_{i_{0}}
$$

is effective nonzero, a contradiction.

q.e.d.

Case 2. Now we deal with the case that $L \neq D^{\prime}$. Then we can write

$$
L=D^{\prime}+L_{0}
$$

where $L_{0}^{m} \in \operatorname{Pic}^{0}(X)$ (The exponent $m$ is there because there might be torsion in $H^{2}(X, \mathbb{Z})$; we take $m$ to kill the denominator of the torsion part). We may in fact assume that $m=1$; otherwise we pass to a finite étale cover $\widetilde{X}$ of $X$ and argue there (the vanishing on $\widetilde{X}$ clearly implies the vanishing on $X$ ). Then the sequence $S$ is modified to

$$
\begin{aligned}
0 \longrightarrow \mathcal{I}(h) \otimes\left(K_{X}+L\right) \longrightarrow K_{X}+L \longrightarrow & \left(K_{X}+L\right) \mid D^{\prime} \\
& =\left(K_{D^{\prime}}+L_{0}\right) \mid D^{\prime} \longrightarrow 0 .
\end{aligned}
$$

Taking cohomology as before, things come down to proving

$$
H^{0}\left(D^{\prime},-L_{0} \mid D^{\prime}\right)=0 .
$$

If $-L_{0} \mid D^{\prime} \neq 0$, then we see as above that $-L_{0} \mid D^{\prime}$ cannot have a nilpotent section. So if $(*)$ fails, then $-L_{0} \mid D^{\prime}$ has a section $s$ such that $s \mid \operatorname{red} D^{\prime}$ has no zeroes, so that $-L_{0} \mid \operatorname{red} D^{\prime}$ is trivial. But then $-L_{0} \mid D^{\prime}$ is trivial. Now let $\alpha: X \longrightarrow A$ be the Albanese map with image $Y$. Then $L_{0}=\alpha^{*}\left(L_{0}^{\prime}\right)$ with some line bundle $L_{0}^{\prime}$ on $A$ which is topologically trivial but not trivial. Since $L_{0} \mid D^{\prime}$ is trivial, we conclude that $\alpha\left(D^{\prime}\right) \neq Y$, and $\alpha\left(D^{\prime}\right)$ is contained in a proper subtorus $B$ of $A$. Now consider the induced map

$$
\beta: X \longrightarrow A / B
$$

and denote its image by $Z$. Then $\beta\left(D^{\prime}\right)$ is a point; on the other hand $D^{\prime}$ is nef, so that $\operatorname{dim} Z=1$ and $D^{\prime}$ consists of multiples of fibers of $\beta$. But this contradicts $D^{\prime 2}=L^{2} \neq 0$.

$$
\text { q.e.d. }
$$

For applications to minimal Kähler 3 -folds, 4.1 is still not good enough, because we need to know the vanishing property $H^{2}\left(X, m K_{X}\right)$ $=0$ on a $\mathbb{Q}$-Gorenstein 3 -fold (with $K_{X}^{2} \neq 0$ ). We would like to set $L=(m-1) K_{X}$ to apply 4.1 but this is no longer a line bundle. This difficulty is overcome by: 
Proposition 4.3. Let $X$ be a normal $\mathbb{Q}$-Gorenstein compact Kähler 3-fold with at most terminal singularities. Let $A$ be a $\mathbb{Q}$-line bundle. Suppose $A$ is nef and $A^{2} \neq 0$. Then

$$
H^{2}\left(X, A+K_{X}\right)=0 .
$$

Proof.

(A) In a first step we show that we may assume $X$ to be $\mathbb{Q}$-factorial. (Actually, in our application in Section 5, it will be clear that we may always assume $X$ to be $\mathbb{Q}$-factorial, so the reader only interested in the applications may skip (A).)

In fact, if $X$ is not $\mathbb{Q}$-factorial, there exists a bimeromorphic map $f: Y \longrightarrow X$ from a normal $\mathbb{Q}$-factorial Kähler space with at most terminal singularities $\left(\left[18,4.5^{\prime}\right]\right)$. Moreover $f$ is an isomorphism in codimension 1 and $f$ is projective since $X$ has only isolated singularities. Now consider the reflexive sheaf

$$
\mathcal{H}=f^{*}\left(\mathcal{O}_{X}(A)\right)^{* *} .
$$

Choose a number $r$ such that $A^{[r]}$ is locally free. Then

$$
\mathcal{H}^{[r]}=f^{*}\left(\mathcal{O}_{X}(r A)\right),
$$

since both sheaves are reflexive and coincide in codimension 1 . Thus $\mathcal{H}$ is nef (as $\mathbb{Q}$-line bundle) with $\mathcal{H}^{2} \neq 0$. Once we know the result in the $\mathbb{Q}$-factorial case, we get

$$
H^{2}\left(Y, \mathcal{H} \hat{\otimes} K_{Y}\right)=0 .
$$

So by the Leray spectral sequence, we only have to show

$$
R^{1} f_{*}\left(\mathcal{H} \hat{\otimes} K_{Y}\right)=0 .
$$

This however follows from [19, 1-2-7]. Actually this citation deals with the algebraic case. However first notice that our statement is local around the isolated singularities of $X$. Now isolated singularities are algebraic by Artin's theorem, i.e., we can realize an open neighborhood of an isolated singularity as an open set in a normal algebraic variety. So locally on $X$ the map $f: Y \longrightarrow X$ can be realized algebraically. Now we can approximate $\mathcal{H}$ by algebraic reflexive sheaves $\mathcal{H}_{k}$ up to high order $k$ and then apply [19, 1-2-7] to get the vanishing $R^{1} f_{*}\left(\mathcal{H}_{k}\right)=0$. This sheaf coincides with $R^{1} f_{*}(\mathcal{H})$ to high order, so $R^{1} f_{*}(\mathcal{H})$ vanishes to high 
order. For $k$ approaching $\infty$, we obtain the vanishing we are looking for.

(B) From now on we assume $X$ to be $\mathbb{Q}$-factorial. We proceed as in the proof of 4.1. First of all choose $r$ such that $A^{[r]}$ is locally free. Then choose a singular metric $h$ with positive curvature current on $A^{[r]}$. Now $\frac{1}{r} h$ is a metric at least on $A \mid X_{\text {reg }}$ with positive curvature current $T$ extending to all of $X$. We argue as in the first part of the proof of 4.1 to obtain the divisor $D$ and the current $R$, however $D$ is only an integral Weil divisor. By the same arguments as in 4.1 we can still reduce the problem to proving

$$
H^{2}\left(D, \mathcal{O}_{D}\left(A+K_{X}\right)\right)=0 .
$$

(Notice that $\mathcal{I}_{D} \otimes \mathcal{O}_{X}\left(A+K_{X}\right)=\mathcal{O}_{X}\left(-D+A+K_{X}\right)$ outside a finite set and that by definition $\mathcal{O}_{D}\left(A+K_{X}\right)=\mathcal{O}_{X}\left(A+K_{X}\right) \mid D$.) Now $D$ is Cohen-Macaulay; here we need in an essential way that locally $X$ is the quotient of a hypersurface by a finite group. To be more detailed, we can write locally $X=V / G$ with $V$ a hypersurface singularity and $G$ a finite group (see e.g., [28]). Let $\pi: V \longrightarrow X$ be the quotient map and let $\hat{D}=\pi^{*}(D)$. If we can prove that $\hat{D}$ is Cohen-Macaulay, then $D$ will be Cohen-Macaulay, too, since this property is $G$-invariant. So we may assume that $X=V$. Now $X$ is (locally) a compound Du Val singularity [28], i.e., a 1-parameter deformation of a 2-dimensional rational double point. Hence we can find a Cartier divisor $H \subset X$ through $x_{0}$ which has just a rational double point at $x_{0}$. Now consider $D \cap H$. This is a Weil $\mathbb{Q}$-Cartier divisor on $H$. Since $x_{0}$ is a quotient singularity of $H$, we can argue as above to see that $D \cap H$ is Cohen-Macaulay. Hence $D$ has a hyperplane section through $x_{0}$ which is Cohen-Macaulay. Thus $D$ is Cohen-Macaulay at $x_{0}$ itself.

Therefore we have by Serre duality

$$
H^{2}\left(D, \mathcal{O}_{D}\left(A+K_{X}\right)\right) \simeq \operatorname{Hom}\left(\mathcal{O}_{D}\left(A+K_{X}\right), \mathcal{O}_{D}\left(K_{D}\right)\right)
$$

Suppose that $H^{2}$ does not vanish. Then we obtain a nonzero homomorphism $s: \mathcal{O}_{D}\left(A+K_{X}\right) \longrightarrow K_{D}$. This $s$ must be generically nonzero. In fact, $D$ is generically Gorenstein. Hence $\mathcal{O}_{D}\left(K_{D}\right)_{x}$ is isomorphic to an ideal in $\mathcal{O}_{X, x}$ for all $x$, in particular $K_{D}$ has no torsion sections, $D$ being Cohen-Macaulay; see [10] in the algebraic case. Let $X_{0}$ be the regular part of $X$, this means that we eliminate a finite set from $X$, all singularities being terminal. Let denote $D_{0}=D \cap X_{0}$ and let $s_{0}=s \mid X_{0}$. 
Then by adjunction we have

$$
0 \neq s_{0} \in H^{0}\left(D_{0}, \mathcal{O}_{D}(-A+D)\right) .
$$

From now on we argue as in 4.1 just working on $X_{0}$ instead of $X$. The only exceptions are calculations of intersection numbers and Hodge index arguments. Here we still need to argue on $X$ - we do not have any problems with singularities since all divisors are $\mathbb{Q}$-Cartier. q.e.d.

\section{The case $K_{X}^{2}=0, K_{X} \neq 0$}

The second ingredient for the proof of the abundance theorem for Kähler threefolds is the following weak analogue of 4.3 in case $K_{X}^{2}=0$ (however one should have in mind that we are dealing with a case which does not exist a posteriori).

Theorem 5.1 Let $X$ be a normal compact Kähler threefold with at most terminal singularities such that $K_{X}$ is nef. Suppose that $K_{X}^{2}=0$, $K_{X} \neq 0$, and that $X$ is simple and not Kummer. Then

$$
h^{2}\left(X, m K_{X}\right) \leq 1
$$

for all $m \in \mathbb{N}$.

As already mentioned the essential property derived from $X$ being simple non-Kummer is that $\pi_{1}(X)$ is finite [3].

\subsection{Start of the proof}

Using Kawamata's $\mathbb{Q}$-factorialisation theorem (compare with the proof of 4.3), we may assume that $X$ is $\mathbb{Q}$-factorial. Suppose $h^{2}\left(X, m K_{X}\right) \geq 2$. Using Serre duality we get - following Miyaoka and Shepherd-Barron - (many) non-split extensions

$$
0 \longrightarrow K_{X} \longrightarrow \mathcal{E} \longrightarrow m K_{X} \longrightarrow 0
$$

with reflexive sheaves $\mathcal{E}$ of rank 2 . We note

$$
c_{1}(\mathcal{E})=(m+1) K_{X}
$$

and

$$
c_{2}(\mathcal{E})=0 .
$$




\subsection{The unstable case}

(5.3.a) Here we will assume that every non-split extension $\mathcal{E}$ as in $(\mathrm{S})$ is $\omega$ - unstable for some fixed Kähler form $\omega$ independent of $\mathcal{E}$. Let $\mathcal{A}_{S} \subset \mathcal{E}$ be the $\omega$-maximal destabilizing subsheaf. Then $\mathcal{A}_{S}$ is a $\mathbb{Q}$-line bundle and we determine a $\mathbb{Q}$-line bundle $\mathcal{B}_{S}$ such that $\mathcal{E} / \mathcal{A}_{S}=\mathcal{I}_{Z} \mathcal{B}_{S}$ with some subspace $Z$ of codimension at least 2 (actually $Z$ is generically (i.e., on the smooth part of $X$ ) locally a complete intersection or finite and supported in $\operatorname{Sing} X)$. Since $K_{X} \neq 0$, we obtain injective maps

$$
\phi_{S}: \mathcal{A}_{S} \longrightarrow m K_{X}
$$

and

$$
\psi_{S}: K_{X} \longrightarrow \mathcal{I B}_{S}
$$

Now there are (up to $\mathbb{C}^{*}$ ) only finitely many maps $\phi_{S}: \mathcal{A}_{S} \longrightarrow m K_{X}$ with some $\mathbb{Q}$-line bundle $\mathcal{A}_{S}$ arising as maximal $\omega$-destabilizing subsheaf for some extension (S). In fact, fix $\phi=\phi_{S}: \mathcal{A} \longrightarrow m K_{X}$. Then by (6.13) there are only finitely many maximal reflexive subsheaves $\mathcal{A}^{\prime} \subset m K_{X}$ such that $\mathcal{A}^{\prime} \not \subset \mathcal{A}$. So we may suppose $\mathcal{A}^{\prime} \subset \mathcal{A}$. Then

$$
\mathcal{A}^{\prime} \cdot \omega^{2}<\mathcal{A} \cdot \omega^{2}
$$

Actually, putting

$$
\epsilon:=\min Y_{j} \cdot \omega^{2},
$$

where the minimum runs over the finitely many irreducible hypersurfaces $Y_{j} \subset X$, we have

$$
\mathcal{A}^{\prime} \cdot \omega^{2} \leq \mathcal{A} \cdot \omega^{2}-\epsilon .
$$

On the other hand, restricting ourselves to $\mathcal{A}^{\prime}$ of the form $\mathcal{A}^{\prime}=\mathcal{A}_{S^{\prime}}$, we have by the destabilizing property

$$
\mathcal{A}^{\prime} \cdot \omega^{2} \geq \frac{c_{1}(\mathcal{E}) \cdot \omega^{2}}{2} .
$$

As there is an integral divisor $\sum \lambda_{j} Y_{j}$ such that

$$
\mathcal{A}^{\prime}=\mathcal{A} \otimes \mathcal{O}_{X}\left(-\sum \lambda_{j} Y_{j}\right)
$$

the finiteness of irreducible hypersurfaces in $X$ gives the finiteness claim, since $(*)$ reads

$$
\left(\mathcal{A}-\sum \lambda_{j} Y_{j}\right) \cdot \omega^{2} \geq \frac{c_{1}(\mathcal{E}) \cdot \omega^{2}}{2}
$$


So we have only finitely many possible maps $\phi$ (up to $\mathbb{C}^{*}$ ). In the same way (by dualizing) we have only finitely many maps $\psi$ (up to $\left.\mathbb{C}^{*}\right)$. In (5.3.b) below we prove that $(\phi, \psi)$ and $(\lambda \phi, \lambda \psi)$ with $\lambda \in \mathbb{C}^{*}$ always define isomorphic extensions $(\mathrm{S})$. Therefore in total $(\lambda \phi, \mu \psi)$ with $\lambda, \mu \in \mathbb{C}^{*}$ define just a 1 -dimensional space of extensions, whence $h^{2}\left(X, m K_{X}\right) \leq 1$.

(5.3.b) We shall now prove that the extension class defining $(\mathrm{S})$ is already determined by $\phi$ and $\psi\left(\operatorname{modulo} \mathbb{C}^{*}\right)$. So take another extension

$$
0 \longrightarrow K_{X} \longrightarrow \mathcal{E}^{\prime} \longrightarrow m K_{X} \longrightarrow 0
$$

with the same destabilizing sheaves $\mathcal{A}$ and $\mathcal{B}$ and with the same morphisms $\phi$ and $\psi$ (the case of $(\phi, \psi),(\lambda \phi, \lambda \psi)$ is exactly the same). Let $D$ be the divisorial part of

$$
\{\phi=0\} \cup\{\psi=0\} \cup \operatorname{Sing}(X) ;
$$

then we obtain a splitting of the sequence (S) over $X \backslash D$ via $\phi$ :

$$
\mathcal{E} \simeq m K_{X} \oplus K_{X} \simeq \mathcal{A} \oplus \mathcal{B}
$$

and an analogous splitting of $\mathcal{E}^{\prime}$ over $X \backslash D$. Observe also that $\mathcal{A}=$ $m K_{X}-D$ and $\mathcal{B}=K_{X}+D$. Thus we obtain an isomorphism

$$
f: \mathcal{E} \longrightarrow \mathcal{E}^{\prime}
$$

on $X \backslash D$ making the two extensions ( $\mathrm{S}$ ) and ( $\left.\mathrm{S}^{\prime}\right)$ isomorphic over $X \backslash D$ :

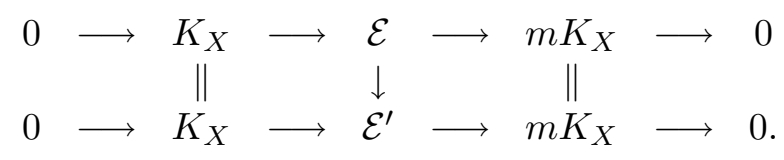

It remains to extend the map $f$ to $X$. Let us notice that we may assume $Z=\emptyset$. In fact let $Z_{1}$ be the codimension 1 part of $Z$. Restricting our two exact sequences describing $\mathcal{E}$ to $D$, we see that (modulo torsion at finitely many points)

$$
\mathcal{A}\left|D=K_{X}\right| D
$$

hence

$$
(m-1) K_{X} \cdot D=D \cdot D
$$


In particular we note that $D \mid D$ is nef, hence $D$ itself is nef. Now (5.3.1) yields

$0=c_{2}(\mathcal{E})=c_{1}(\mathcal{A}) \cdot c_{1}(\mathcal{B})+c_{2}\left(\mathcal{I}_{Z} B\right)=\left(m K_{X}-D\right) \cdot\left(K_{X}+D\right)+Z_{1}=Z_{1}$,

hence $Z_{1}=\emptyset$. In particular $Z \subset \operatorname{Sing} X$ has codimension at least 3 . This shows that we may ignore $Z$ in all our following considerations; in what follows restriction will always that we also divide by torsion. Take a local section $s \in \mathcal{E}(U)$ over a small disc $U$. We need to show that $f(s) \in \mathcal{E}^{\prime}(U)$; a priori we only know $f(s) \in \mathcal{E}^{\prime}(D)(U)$. Let

$$
\kappa: \mathcal{E} \rightarrow m K_{X}, \kappa^{\prime}: \mathcal{E}^{\prime} \rightarrow m K_{X}
$$

and

$$
\lambda: \mathcal{E} \rightarrow \mathcal{B}, \lambda^{\prime}: \mathcal{E}^{\prime} \rightarrow \mathcal{B}
$$

be the canonical maps.

Now consider the exact sequence

$$
0 \longrightarrow \mathcal{E} \stackrel{\kappa \oplus \lambda}{\longrightarrow} m K_{X} \oplus \mathcal{B} \stackrel{\rho}{\longrightarrow} m K_{X} \mid D \longrightarrow 0
$$

Here $\rho(u \oplus v)=u_{D}-\tau\left(v_{D}\right)$, where $\tau: \mathcal{B}_{D} \rightarrow m K_{X} \mid D$ is the canonical sequence arising by restricting our sequences and the maps $\phi$ and $\psi$ to $D$. Analogously for $\mathcal{E}^{\prime}$. Suppose we know

$$
h^{0}\left(D_{c}, \mathcal{O}_{D_{c}}\right)=1
$$

for any connected component $D_{c}$ of $D$. Actually it suffices to know this for $D_{c} \cap \operatorname{reg} X$. Then $\tau^{\prime-1} \circ \tau=a \mathrm{id}$, and of course we can normalize (in the extension class) to $a=1$ (our arguments are local around every individual connected component of $D$ ). Therefore we can construct a diagram

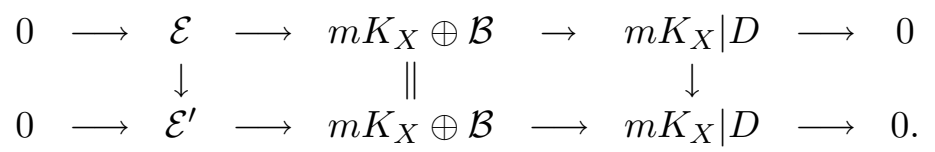

Here the map $f: \mathcal{E} \rightarrow \mathcal{E}^{\prime}$ is defined only on $X \backslash D$ and meromorphic on $X$, the left square is commutative on $X \backslash D$ and the right square is commutative on $X$. It is immediately checked that $m K_{X}\left|D \rightarrow m K_{X}\right| D$ is the identity (consider images of elements $u \oplus 0$ ), hence $f(s) \in \mathcal{E}^{\prime}(U)$ and thus $f$ extends to a global isomorphism making the two extensions isomorphic. 
(5.3.c) It remains to prove $(+)$ and we may assume $D=D_{c}$ for simplicity of notations. So suppose that $h^{0}\left(D, \mathcal{O}_{D}\right) \geq 2$, resp. that $h^{0}\left(D \cap \operatorname{reg} X, \mathcal{O}_{D \cap \operatorname{reg} X}\right) \geq 2$. Since $H^{1}\left(X, \mathcal{O}_{X}\right)=0$, we obtain

$$
H^{1}\left(X, \mathcal{O}_{X}(-D)\right) \neq 0 \text {. }
$$

Let $X_{0}$ be the regular part of $X$ and $D_{0}=D \mid X_{0}$. Since the singularities of $X$ are at most finite,

$$
H^{1}\left(X_{0}, \mathcal{O}\left(-D_{0}\right)\right)=H^{1}(X, \mathcal{O}(-D)) \neq 0 .
$$

Hence $\operatorname{Ext}^{1}\left(\mathcal{O}_{X_{0}}, \mathcal{O}\left(-D_{0}\right)\right) \neq 0$, and therefore there exists a non-split extension

$$
0 \longrightarrow \mathcal{O}_{X_{0}} \longrightarrow \mathcal{F}_{0} \longrightarrow \mathcal{O}\left(D_{0}\right) \longrightarrow 0,
$$

with a locally free sheaf $\mathcal{F}_{0}$ over $X_{0}$. Now $\mathcal{F}_{0}$ has a unique reflexive extension to $X$ : consider a singular point $x_{0} \in X$ and let $U$ be a Stein neighborhood of $x_{0}$. Then

$$
H^{1}\left(U \backslash x_{0}, \mathcal{O}\left(-D_{0}\right)\right)=H^{1}(U, \mathcal{O}(-D))=0,
$$

hence $\left(\mathrm{E}_{0}\right)$ splits over $U \backslash x_{0}$ :

$$
\mathcal{F}_{0} \simeq \mathcal{O} \oplus \mathcal{O}_{U \backslash x_{0}}\left(D_{0}\right) \text {. }
$$

Hence $\mathcal{F}_{0}$ extends to a reflexive sheaf $\mathcal{F}$. Moreover $\left(E_{0}\right)$ extends to

$$
0 \longrightarrow \mathcal{O}_{X} \longrightarrow \mathcal{F} \longrightarrow \mathcal{O}_{X}(D) \longrightarrow 0 .
$$

In particular $\operatorname{Ext}^{1}\left(\mathcal{O}_{X}(D), \mathcal{O}_{X}\right) \neq 0$. This is easily seen to be equivalent to

$$
\operatorname{Ext}^{1}\left(K_{X}+\mathcal{O}_{X}(D), \mathcal{O}_{X}(D)\right) \neq 0
$$

hence $H^{2}\left(X, K_{X}+\mathcal{O}_{X}(D)\right) \neq 0$ by Serre duality. Thus

$$
D^{2}=0
$$

by (4.3).

We observe $c_{1}(\mathcal{F})^{2}=c_{2}(\mathcal{F})=0$, therefore $\mathcal{F}$ cannot be $\omega$-stable. So let $\mathcal{A}^{\prime}$ be the maximal $\omega$-destabilizing subsheaf, and we obtain as before for $\mathcal{E}$ a sequence

$$
0 \longrightarrow \mathcal{A}^{\prime} \longrightarrow \mathcal{F} \longrightarrow \mathcal{I}_{Z^{\prime}} \mathcal{B}^{\prime} \longrightarrow 0
$$


where $Z^{\prime}$ has generically dimension 1 or is contained in the singular locus of $X$. As before, $\mathcal{A}^{\prime} \subset \mathcal{O}_{X}(D)$ and $\mathcal{O}_{X} \subset \mathcal{B}^{\prime}$ so that there is an effective divisor $D^{\prime}$ such that $\mathcal{B}^{\prime}=\mathcal{O}_{X}\left(D^{\prime}\right)$ and $\mathcal{A}^{\prime}=\mathcal{O}_{X}\left(D-D^{\prime}\right)$. By (E) and the fact that $a(X)=0$ and $H^{1}\left(X, \mathcal{O}_{X}\right)=0$, we deduce that $h^{0}(X, \mathcal{F})=2$. Hence $(\mathrm{F})$ yields

$$
h^{0}\left(X, \mathcal{A}^{\prime}\right)=1
$$

So we can write $\mathcal{A}^{\prime}=\mathcal{O}_{X}(E)$ with an effective divisor $E$. Thus $D=$ $D^{\prime}+E$. By restricting (E) and (F) to $D^{\prime}$, we obtain $\mathcal{A}^{\prime}\left|D^{\prime}=E\right| D^{\prime}=\mathcal{O}_{D^{\prime}}$ and that the 1-dimensional part $Z_{1}^{\prime}$ of $Z^{\prime}$ is empty, so that $Z^{\prime}$ is at most finite. Alternatively, we calculate

$$
0=c_{2}(\mathcal{F})=E \cdot D^{\prime}+Z_{1}^{\prime}
$$

and conclude together with the observation that $D^{\prime} \mid D^{\prime}$ and thus $D^{\prime}$ is nef.

So we have a decomposition $D=D^{\prime}+E$ with $D$ and $D^{\prime}$ nef. By instability, $E \neq 0$. Having in mind that $D$ is connected, we are going to prove that $D, D^{\prime}$ and $E$ are proportional (even numerical proportionality would be sufficient, which in our situation ( $X$ simply connected with $a(X)=0)$ gives equality). Assuming this proportionality for the moment, we obtain $D^{\prime}=a E$ and $D=(a+1) E$. Since $\mathcal{A}^{\prime}$ destabilizes, we have $a \leq 1$. By restricting the sequence $(\mathrm{E})$ and $(\mathrm{F})$ to $D^{\prime}$ we obtain:

$$
\mathcal{O}_{D^{\prime}}(E)=\mathcal{O}_{D^{\prime}}
$$

up to torsion. For the simplicity of notation we suppress the torsion and agree, when taking a restriction, that we also divide by the torsion. We may assume that $h^{0}\left(D^{\prime}, \mathcal{O}_{D^{\prime}}\right)=1$. In fact, if $h^{0}\left(D^{\prime}, \mathcal{O}_{D^{\prime}}\right) \geq 2$, then we substitute $D$ by $D^{\prime}$ and argue as before; of course this procedure has to terminate after finitely many steps. So $h^{0}\left(D^{\prime}, \mathcal{O}_{D^{\prime}}\right)=1$ and consequently

$$
h^{0}\left(D^{\prime}, N_{D^{\prime} / D}^{* \mu}\right) \neq 0
$$

for some positive integer; hence $h^{0}\left(D^{\prime}, N_{D^{\prime} / X}^{* \mu}\right) \neq 0$. (We may neglect the torsion, because we may compute over $X_{0}$ ).

Now this non-vanishing implies that $\mu D^{\prime} \mid D_{0}$ is trivial, where $D_{0}$ is the reduction of $D^{\prime}$. In fact, let $\mu D^{\prime}=\sum a_{i} Y_{i}$ and take a nonzero section 
$s$ of $\mathcal{O}_{\mu D^{\prime}}\left(\mu D^{\prime}\right)$. Let $\widetilde{D}=\sum b_{i} Y_{i} \subset \mu D^{\prime}$ be the maximal subdivisor such that $s \mid \widetilde{D}=0$. Introducing $c_{i}=a_{i}-b_{i} \geq 0$, we obtain a section

$$
s^{\prime} \in H^{0}\left(D_{0}, \mathcal{O}_{D_{0}}\left(-\sum c_{i} Y_{i}\right)\right)
$$

such that $s^{\prime} \mid Y_{i} \neq 0$ for all $i$. Fix a Kähler form $\omega$ and let $\alpha_{i j}=Y_{i} \cdot Y_{j} \cdot \omega$ for $i \neq j$. Then we obtain for all $j$

$$
-\sum_{i} c_{i} Y_{i} \cdot Y_{j} \cdot \omega=-\sum c_{i} \alpha_{i j} \geq 0
$$

Since $D^{2}=0$ and $D$ is nef, we have $D \cdot Y_{j}=0$ for all $j$ and therefore

$$
Y_{j}^{2}=-\frac{1}{a_{j}} \sum_{i \neq j} a_{i} Y_{i} \cdot Y_{j}
$$

so that we arrive at the inequalities (for each $j$ )

$$
\sum_{i \neq j} a_{i} c_{j} \alpha_{i j} \geq \sum_{i \neq j} a_{j} c_{i} \alpha_{i j}
$$

By simple algebraic considerations this is only possible if we have always equality. This means that the divisor $D^{*}=\sum c_{i} Y_{i}$ fulfills $D^{*} \cdot Y_{j}=0$ for all $j$. Hence $D^{*}$ is nef, and the proportionality arguments below shows that $D^{*}=c D^{\prime}$ for some positive number $c$. Because of the nonvanishing of $s^{\prime}$ on $Y_{j}, D^{*} \mid Y_{j}$ is trivial, hence $D^{*} \mid D_{0}$ is trivial. Suppose for the moment that $\mu=1$. Then $D^{*} \subset D^{\prime}$ so that $c<1$ and in total we have $D^{*} \mid D_{0}$ trivial, and $D^{*} \mid D^{*}$ torsion by $(*)$. Hence $[25,4.1]$ says that $D^{*} \mid D^{*}$ is trivial. Now the exact sequence

$$
0 \longrightarrow \mathcal{O}_{X} \longrightarrow \mathcal{O}_{X}\left(D^{*}\right) \longrightarrow \mathcal{O}_{X}\left(D^{*}\right) \mid D^{*} \longrightarrow 0
$$

implies by $(*)$ - keeping in mind $H^{1}\left(X, \mathcal{O}_{X}\right)=0$ - that $h^{0}\left(X, \mathcal{O}_{X}\left(D^{\prime}\right)\right)$ $\geq 2$, contradicting $a(X)=0$. So we are left with the case $\mu \geq 2$. We deal with $\mu=2$ and leave the trivial modifications in the general case to the reader. The difficulty here is that possibly $c>1$ so that $D^{\prime} \subset D^{*}$, otherwise we conclude as before. At least we know that $D^{*} \subset \mu D^{\prime}$ and we are going to show that $\mu E \mid \mu D^{\prime}$ is trivial; then we are done. This does not follows directly from restricting $(\mathrm{E})$ and $(\mathrm{F})$; instead we take $S^{2}$ and obtain an injection $\mathcal{O}_{X}(2 E) \longrightarrow S^{2}(\mathcal{F})$. Restricting to $2 D^{\prime}$, we obtain a nonzero map $\mathcal{O}_{2 D^{\prime}}(2 E) \rightarrow \mathcal{F} \mid 2 D^{\prime}$. Then either the induced map 
$\left.\mathcal{O}_{2 D^{\prime}}(2 E) \rightarrow \mathcal{O}_{D}(D)\right)$ is nonzero; this implies $H^{0}\left(D^{\prime}, \mathcal{O}_{D^{\prime}}\left(-D^{\prime}\right)\right) \neq 0$ so that $h^{0}\left(X, \mathcal{O}_{2 D^{\prime}}\right)>1$ and we may take $D=2 D^{\prime}$ whence $\mu=1$. Or this map vanishes; then we get a nonzero map $\mathcal{O}_{2 D^{\prime}}(2 E) \rightarrow \mathcal{O}_{2 D^{\prime}}$. This map is an isomorphism and settles our claim.

(5.3.d) It remains to settle the proportionality. After passing to a desingularization this comes down to proving the following statement: Let $X$ be compact Kähler, $A=\sum_{i=1}^{s} a_{i} Y_{i}$ and $B=\sum_{i=1}^{s} b_{i} Y_{i}$ be effective divisors, $a_{i}$ and $b_{i}$ positive, with connected supports. Suppose that $A$ and $B$ are nef, and that $A \cdot Y_{i}=B \cdot Y_{i}=0$ for all $i$ (in particular $A^{2}=B^{2}=A \cdot B=0$ ). Then $A=c B$ (not only for numerical equivalence). Observe that if $X$ is a surface, then this is nothing than Zariski's lemma, which is usually formulated for fibers of maps to curves, but which works in this context; therefore the claim also follows for projective manifolds by taking hyperplane sections and applying Lefschetz. If $X$ is merely Kähler, then we consider the vector space $V \subset H^{2}(X, \mathbb{R})$ generated by the classes of the hypersurfaces $Y_{i} \subset X$. Let $W$ be the direct $\operatorname{sum} \bigoplus \mathbb{R} \cdot Y_{i}$; and let $Q$ be the bilinear form

$$
Q\left(Y_{i}, Y_{j}\right)=-Y_{i} \cdot Y_{j} \cdot \omega
$$

In this situation we apply [1, Lemma I.2.10] to conclude.

q.e.d.

5.4 The stable case. By 5.3 we are reduced to the case that some extension $\mathcal{E}$ is $\omega$-stable for some Kähler form $\omega$. By (5.2.1) and (5.2.2) we have in particular

$$
c_{1}^{2}(\mathcal{E}) \cdot \omega=4 c_{2}(\mathcal{E}) \cdot \omega
$$

which implies that $\mathcal{E}$ is projectively flat, at least on the regular part $X_{0}$. In fact, this is well-known if $X$ is smooth and $\mathcal{E}$ is locally free. But the proof generalizes to our case since the singularities of $X$ and $\mathcal{E}$ are in codimension at least 3 . Now we follow the arguments in $[22, \mathrm{p}$. $113 / 114]$.

Assume first that the degree of finite étale covers of $X_{0}$ is bounded: $\pi_{1}^{\text {alg }}\left(X_{0}\right)$ is finite. After performing a finite étale cover, we may assume $\pi_{1}^{\text {alg }}\left(X_{0}\right)=0$. Since $\mathcal{E} \mid X_{0}$ is projectively flat, $\mathcal{E}^{*} \otimes \mathcal{E} \mid X_{0}$ is hermitian flat and therefore given by a unitary representation $\rho$ of $\pi_{1}\left(X_{0}\right)$. Since $\rho\left(\pi_{1}\left(X_{0}\right)\right)$ is residually finite, it follows that $\rho$ is trivial, hence $\mathcal{E}^{*} \otimes \mathcal{E}$ is trivial. This implies, using the exact sequence

$$
0 \longrightarrow K_{X} \longrightarrow \mathcal{E} \longrightarrow m K_{X} \longrightarrow 0
$$


and dualizing that (over $\left.X_{0}\right) h^{0}\left(\mathcal{E}^{*} \otimes K_{X}\right)=0$, that $h^{0}\left(\mathcal{E}^{*} \otimes m K_{X}\right) \geq 4$ and that therefore $h^{0}\left((m-1) K_{X}\right) \geq 3$. This contradicts $a(X)=0$.

If $\pi_{1}^{\text {alg }}\left(X_{0}\right)$ is infinite, we just take over the arguments of [22, p. 114]: since the local fundamental groups of $X$ at the singularities are finite, any finite étale cover $h$ of $X_{0}$ of sufficiently large degree extends to a covering $h: \widetilde{X} \rightarrow X$ which can be written in the form $h=g \circ f$, where $f: \widetilde{X} \rightarrow X^{\prime}$ is étale and $g: X^{\prime} \rightarrow X$ is étale outside the singular locus. Therefore $\pi_{1}\left(X^{\prime}\right)$ is infinite, contradicting the fact that $X^{\prime}$ is simple non-Kummer.

q.e.d.

\section{The inequality $K_{X} \cdot c_{2}(X) \geq 0$}

The aim of this section is to prove:

Theorem 6.1. Let $X$ be a minimal Kähler 3-fold with $a(X)=0$. Then $K_{X} \cdot c_{2}(X) \geq 0$.

This inequality is an important step in the proof of abundance for Kähler threefolds. In the projective case, it follows from Miyaoka's inequality $K_{X}^{2} \leq 3 c_{2}(X)$ which in turn is a consequence of his generic nefness theorem for the cotangent bundle (relying on char $p$ methods).

The rest of this section consists of the proof of 6.1 together with some auxiliary propositions $(6.9,6.10,6.12 / 6.13)$.

\subsection{Reduction to the unstable case}

Suppose that there is a sequence $\left(\omega_{j}\right)$ of Kähler metrics converging in $H^{2}(X, \mathbb{R})$ to $K_{X}$ such that $T_{X}$ is $\omega_{j}$-stable for all $j$. Then we have

$$
c_{1}^{2}(X) \cdot \omega_{j} \leq 3 c_{2}(X) \cdot \omega_{j}
$$

for all $j$ by Proposition 6.9. Taking limits, we obtain

$$
K_{X}^{3} \leq 3 K_{X} \cdot c_{2}(X)
$$

hence our claim results from $K_{X}^{3}=0$.

So from now on we shall assume that $T_{X}$ is $\omega$-unstable for all $\omega$ near $K_{X}\left(\right.$ in $\left.H^{2}(X, \mathbb{R})\right)$. 


\subsection{The setup}

Let $\mathcal{S}_{\omega} \subset T_{X}$ be the maximal destabilizing subsheaf with respect to $\omega$. Let $r$ denote its rank. Then by Corollary 6.13 below, there are only finitely many choices for $\mathcal{S}_{\omega}$, hence there exists an open set in the Kähler cone of $X$ having $K_{X}$ as boundary point such that $\mathcal{S}_{\omega}$ does not depend on $[\omega]$ for $[\omega] \in U$. We shall write

$$
S=\operatorname{det} \mathcal{S}
$$

and let $Q=T_{X} / \mathcal{S}$, a torsion free sheaf of rank 1 or 2 . We notice

$$
c_{1}(Q)=-K_{X}-S
$$

and, if $r=1$,

$$
c_{2}(Q)=c_{2}(X)+S \cdot\left(K_{X}+S\right) .
$$

$c_{3}(Q)$ will be irrelevant for us. The instability of $T_{X}$ gives

$$
S \cdot \omega^{2} \geq \frac{-K_{X} \cdot \omega^{2}}{3},
$$

for $\omega \in U$ in case $r=1$ and

$$
\frac{S \cdot \omega^{2}}{2} \geq \frac{-K_{X} \cdot \omega^{2}}{3}
$$

in case $r=2$. We claim

$$
K_{X}^{2} \cdot S=0
$$

In fact, (6.3.3) resp. (6.3.3a) gives in the limit $K_{X}^{2} \cdot S \geq 0$. Since we may assume $K_{X}^{2} \neq 0$, the tangent sheaf $T_{X}$ is $K_{X}$-semi-stable by Enoki [11]. This implies $K_{X}^{2} \cdot S \leq 0$, hence (6.3.4) follows.

The next lemma is a general statement on Kähler 3 -folds with $a(X)$ $=0$, independent from our setup.

Lemma 6.4. Let $X$ be a normal compact Kähler 3 -fold. Let $A$ and $B$ be $\mathbb{Q}$-line bundles on $X$ and let $A$ be nef with $A^{2} \neq 0$. If $A^{2} \cdot B=0$, then $A \cdot B^{2} \leq 0$.

Proof. By passing to a desingularization we may assume $X$ smooth. Fix a Kähler class $\omega$ and apply (2.5) with $\alpha=c_{1}(B), \beta=K_{X}+\varepsilon \omega$ and $\gamma=K_{X}$. Then expand in terms of powers of $\varepsilon$ to obtain the claim. 
Corollary 6.4.a. In our setup (6.3) we have $K_{X} \cdot S^{2} \leq 0$ if $K_{X}^{2}=0$.

Proof. This follows from 6.4 via 6.3.4.

q.e.d.

Lemma 6.4 is of course not true in case $A^{2}=0$. Thus in order to obtain (6.4.a) also in case $K_{X}^{2}=0$ we need more specific arguments:

Lemma 6.5. Let $X$ be a simply connected minimal Kähler 3-fold with $a(X)=0$ and $K_{X}^{2}=0$. Let $L$ be a $\mathbb{Q}$-line bundle on $X$. Then $K_{X} \cdot L^{2} \leq 0$.

Proof. Assume that $K_{X} \cdot L^{2}>0$. If a positive integer $c$ satisfies the following condition:

$$
2 c^{2} K_{X} \cdot L^{2}>-K_{X} \cdot c_{2}(X)
$$

then by Riemann-Roch we easily get asymptotically

$$
\chi\left(X, m K_{X}+c L\right) \sim m .
$$

Observe also that $(*)$ is satisfied for large $c$ since $K_{X} \cdot L^{2}>0$ by assumption. So let us fix such a number $c$. Then we conclude

$$
h^{2}\left(m K_{X}+c L\right) \geq C m .
$$

In fact, otherwise $h^{0}\left(m K_{X}+c L\right) \geq C m$ by $(*)$, contradicting $a(X)=0$.

Now, as in Section 5, we obtain "many" extensions

$$
0 \longrightarrow K_{X}+c L \longrightarrow \mathcal{E} \longrightarrow m K_{X} \rightarrow 0 \text {. }
$$

Observe that $\mathcal{E}$ cannot be $\omega$-stable for $\omega$ near $K_{X}$. In fact, in that case we had

$$
c_{1}(\mathcal{E})^{2} \cdot \omega \leq 4 c_{2}(\mathcal{E}) \cdot \omega,
$$

hence $c_{1}(\mathcal{E})^{2} \cdot K_{X} \leq 4 c_{2}(\mathcal{E}) \cdot K_{X}$ in the limit. This comes down to

$$
c^{2} K_{X} \cdot L^{2} \leq 0
$$

contradicting our assumption.

We proceed exactly in the same way as in Section 5, introducing the divisors $D_{m}$, and now $(* *)$ and the arguments in Section 5 yield

$$
h^{0}\left(\mathcal{O}_{D_{m}}\right) \geq C m
$$


for large $m$. On the other hand,

$$
(m-1) K_{X}+c L^{*}\left|D_{m}=D_{m}\right| D_{m},
$$

again referring to Section 5 , hence for large $m$, the normal bundle $N_{D_{m}}$ gets more and more "nef". However, to have many functions on $D_{m}$ means to have a tendency to negativity for the normal bundle. So we will show that $(+)$ and $(++)$ are contradictory. By passing to a subsequence - having in mind that $X$ carries only finitely many irreducible hypersurfaces - we can suppose the following:

$$
D_{m}=\sum_{i=1}^{s} a_{m, i} Y_{i}+\sum_{s+1}^{t} a_{j} Y_{j}
$$

where $a_{m, i}<a_{m+1, i}$ and the $a_{j}$ are independent of $m$. Put $R=$ $\sum_{s+1}^{t} a_{j} Y_{j}$ and $Y=\sum_{1}^{s} Y_{i}$. Then

$$
N_{D_{m} / D_{m+1}}^{*}=\left(\sum_{i=1}^{s} a_{m, i} Y_{i}+R\right) \mid Y .
$$

Since by $(+), h^{0}\left(N_{D_{m} / D_{m+1}}^{*}\right)>0$, the sequence of divisors $-\sum a_{m, i} Y_{i} \mid Y$, suitably normalized, converges to an effective nonzero divisor on $Y$. Thus $N_{D_{m} \mid X}^{*} \mid Y$, suitably normalized converges to an effective nonzero divisor on $Y$. On the other hand, its dual is nef by $(++)$. This is a contradiction.

q.e.d.

Corollary 6.5.a. In our setup (6.3) we have $K_{X} \cdot S^{2} \leq 0$.

\subsection{The Case: $\operatorname{rk} \mathcal{S}=1$ and $Q$ stable}

By " $Q$ stable" we mean that there is a sequence of Kähler forms $\left(\omega_{j}\right)$ converging to $K_{X}$ (as classes) such that $Q$ is $\omega_{j}$-stable for all $j$. Then by Proposition 6.9 we have

$$
c_{1}^{2}(Q) \cdot \omega_{j} \leq 4 c_{2}(Q) \cdot \omega_{j}
$$

hence

$$
c_{1}^{2}(Q) \cdot K_{X} \leq 4 c_{2}(Q) K_{X}
$$

Putting in (6.3.1) and (6.3.2) we obtain

$$
K_{X} \cdot\left(K_{X}+S\right)^{2} \leq\left(4 c_{2}(X)+4 S \cdot\left(K_{X}+S\right)\right) \cdot K_{X},
$$


which in turn yields

$$
K_{X} \cdot c_{2}(X) \geq-\frac{3}{4} K_{X} \cdot S^{2}
$$

Thus 6.4.a gives $K_{X} \cdot c_{2}(X) \geq 0$.

\subsection{The Case: rk $\mathcal{S}=1$ and $Q$ is unstable}

After the previous case it is clear what unstable has to mean: $Q$ is $\omega$-unstable for all $\omega$ near $K_{X}$. Then we obtain a destabilizing sequence

$$
0 \longrightarrow L_{1} \longrightarrow Q \longrightarrow \mathcal{I}_{B} L_{2} \longrightarrow 0
$$

where $L_{i}$ are reflexive of rank 1 and $\operatorname{dim} B \leq 1$. This sequence is - as usual - independent of $\omega$, if $\omega$ is sufficiently near to $K_{X}$ and contained in a suitable open set $U$ as in (6.3). We first claim

$$
K_{X}^{2} \cdot L_{1} \leq 0
$$

To verify this, let $\mathcal{R}$ be the cokernel of

$$
T_{X} \longrightarrow \mathcal{I}_{B} L_{2} \longrightarrow 0 .
$$

Then we have an exact sequence

$$
0 \longrightarrow S \longrightarrow \mathcal{R} \longrightarrow L_{1} \longrightarrow 0 \text {. }
$$

Of course we may assume $K_{X}^{2} \neq 0$. Then by Enoki [En87], $T_{X}$ is $K_{X^{-}}$ semi-stable, hence

$$
c_{1}(\mathcal{R}) \cdot K_{X}^{2} \leq 0
$$

This implies $K_{X}^{2} \cdot\left(L_{1}+S\right) \leq 0$ by the last exact sequence. Now (6.3.4) gives our claim (6.7.1).

Next we show

$$
K_{X} \cdot L_{1}=0
$$

In fact, the destabilizing property for $L_{1}$ reads

$$
L_{1} \cdot \omega^{2} \geq \frac{c_{1}(Q) \cdot \omega^{2}}{2}
$$

hence

$$
L_{1} \cdot K_{X}^{2} \geq \frac{c_{1}(Q) \cdot K_{X}^{2}}{2}=\frac{1}{2}\left(-K_{X}-S\right) \cdot K_{X}^{2}=0
$$


We now conclude by (6.7.1).

Since $c_{1}(Q) \cdot K_{X}^{2}=0$, we also have

$$
K_{X}^{2} \cdot L_{2}=0 .
$$

Thus Lemma 6.4 applies:

$$
K_{X} \cdot L_{i}^{2} \leq 0
$$

for $i=1,2$.

The final preparation is

$$
K_{X} \cdot L_{1} \cdot L_{2}=\frac{1}{2}\left(K_{X} \cdot S^{2}-K_{X} \cdot L_{1}^{2}-K_{X} \cdot L_{2}^{2}\right) .
$$

This follows from the two equations

$$
K_{X} \cdot c_{1}^{2}(Q)=K_{X} \cdot\left(L_{1}+L_{2}\right)^{2}
$$

and

$$
K_{X} \cdot c_{1}^{2}(Q)=K_{X} \cdot\left(K_{X}+S\right)^{2}=K_{X} \cdot S^{2} .
$$

After all these preparations we conclude using (6.7.4) as follows:

$$
\begin{aligned}
K_{X} \cdot c_{2}(X) & =K_{X} \cdot c_{2}(Q)+K_{X} \cdot S \cdot c_{1}(Q) \\
& =K_{X} \cdot c_{2}\left(\mathcal{I}_{B}\right)+K_{X} \cdot L_{1} \cdot L_{2}-K_{X} \cdot S^{2} \\
& =K_{X} \cdot c_{2}\left(\mathcal{I}_{B}\right)+\frac{K_{X} \cdot S^{2}}{2}-\frac{K_{X} \cdot L_{1}^{2}}{2}-\frac{K_{X} \cdot L_{2}^{2}}{2}-K_{X} \cdot S^{2} .
\end{aligned}
$$

Since $K_{X} \cdot c_{2}\left(\mathcal{I}_{B}\right) \geq 0$ by the nefness of $K_{X}$, we conclude by virtue of (6.7.4) and Lemma 6.4.

\subsection{The Case: $\operatorname{rk} \mathcal{S}=2$}

In this case we consider the maximal destabilizing subsheaf $Q^{*} \subset \Omega_{X}^{1}=$ $\left(T_{X}\right)^{*}$. Here it is convenient to switch completely the notations: we denote the maximal destabilizing subsheaf of $\Omega_{X}^{1}$ again by $\mathcal{S}$ and let $Q$ denote the quotient. Then

$$
c_{1}(Q)=K_{X}-S
$$

and

$$
c_{2}(Q)=c_{2}(X)-S \cdot\left(K_{X}-S\right) .
$$

Now (6.3) yields $K_{X}^{2} \cdot S=0$. Applying again 6.4 gives $K_{X} \cdot S^{2} \leq 0$. Now (6.6) and (6.7) run in completely the same way; notice that some minus signs are irrelevant because $K_{X}^{2} \cdot S=0$. 
Proposition 6.9. Let $X$ be a normal compact Kähler $n$-fold with codim $\operatorname{Sing}(X) \geq 3$. Suppose $a(X)=0$. Let $\omega$ be a Kähler form on $X$ and $\mathcal{E}$ a torsion free coherent sheaf on $X$ of rank $r \geq 2$. If $\mathcal{E}$ is $\omega$-stable, then

$$
c_{1}^{2}(\mathcal{E}) \cdot \omega^{n-2} \leq \frac{2 r}{r-1} c_{2}(\mathcal{E}) \cdot \omega^{n-2} .
$$

Proof. For simplicity of notations set $\mu=\frac{2 r}{r-1}$.

(1) First we reduce the problem to the case "E $\mathcal{E}$ reflexive". So suppose we know the assertion for reflexive sheaves and let $\mathcal{E}$ be torsion free. Then we consider the quotient sheaf

$$
Q=\mathcal{E}^{* *} / \mathcal{E},
$$

which is supported on a complex subspace $Z \subset X$ of codimension at least 2. Now

$$
c_{2}(Q)=-m c_{2}\left(\mathcal{I}_{Z}\right),
$$

for some positive $m$, and $c_{2}\left(\mathcal{I}_{Z}\right)$ is an effective cycle supported on $Z$, hence

$$
\omega^{n-2} \cdot c_{2}(Q) \leq 0 .
$$

Now $c_{2}\left(\mathcal{E}^{* *}\right)=c_{2}(\mathcal{E})+c_{2}(Q)$, hence $(*)$ implies

$$
c_{2}(\mathcal{E}) \omega^{n-2} \geq c_{2}\left(\mathcal{E}^{* *}\right) \cdot \omega^{n-2} .
$$

Notice that $\mathcal{E}^{* *}$ is stable because $\mathcal{E}$ is $([20$, V.7.7]), hence by our assumption

$$
c_{1}^{2}\left(\mathcal{E}^{* *}\right) \omega^{n-2} \leq \mu c_{2}\left(\mathcal{E}^{* *}\right) \cdot \omega^{n-2} .
$$

Since $c_{1}\left(\mathcal{E}^{* *}\right)=c_{1}(\mathcal{E})$, the inequality $(* *)$ implies our claim follows.

(2) From now on we shall assume $\mathcal{E}$ reflexive. Choose a desingularization $\pi: \hat{X} \longrightarrow X$ by a sequence of blow-ups whose centers all lie over the singularities of $X$ and $\mathcal{E}$. Moreover we may assume that $\hat{\mathcal{E}}=\pi^{*}(\mathcal{E})^{* *}$ is locally free (see [14]). Let $\hat{\omega}=\pi^{*}(\omega)$. By definition of Kähler forms on singular spaces $\hat{\omega}$ - which a priori exists only on a Zariski open part of $\hat{X}$ - extends to a semipositive $(1,1)$-form on all of $\hat{X}$. We claim

$$
\mathcal{E} \text { is } \hat{\omega} \text { - stable. }
$$

Indeed, assume we have a subsheaf $\hat{\mathcal{S}} \subset \hat{\mathcal{E}}$ of rank $s$ with

$$
\frac{c_{1}(\hat{\mathcal{S}}) \cdot \hat{\omega}^{n-1}}{s} \geq \frac{c_{1}(\hat{\mathcal{E}}) \cdot \hat{\omega}^{n-1}}{r} .
$$


Then consider

$$
\mathcal{S}=\pi_{*}(\hat{\mathcal{S}}) \subset \pi_{*}(\hat{\mathcal{E}}) .
$$

Since $\pi_{*}(\hat{\mathcal{E}})$ is torsion free and since $\mathcal{E}$ is reflexive, we have $\pi_{*}(\hat{\mathcal{E}}) \subset \mathcal{E}$, hence $\mathcal{S} \subset \mathcal{E}$. Now

$$
c_{1}(\hat{S}) \cdot \hat{\omega}^{n-1}=c_{1}\left(\pi_{*}(\hat{\mathcal{S}})\right) \cdot \omega^{n-1}=c_{1}(\mathcal{S}) \cdot \omega^{n-1},
$$

and

$$
c_{1}(\hat{\mathcal{E}}) \cdot \hat{\omega}^{n-1}=c_{1}(\mathcal{E}) \cdot \omega^{n-1}
$$

hence

$$
\frac{c_{1}(\mathcal{S}) \cdot \omega^{n-1}}{s} \geq \frac{c_{1}(\mathcal{E}) \cdot \omega^{n-1}}{r},
$$

contradicting the $\omega$-stability of $\mathcal{E}$. This proves $(+)$.

Now $\hat{\omega}$ has the disadvantage not to be a Kähler form, but it is on the boundary of the Kähler cone. To circumvent this difficulty, let $E_{i}$ denote the exceptional components of the exceptional set of $\pi$, then we can chose $a_{i}<0$, such that $E:=\sum a_{i} E_{i}$ is $\pi$-ample. Thus

$$
\hat{\omega}_{\epsilon}:=\hat{\omega}+\epsilon E
$$

is a Kähler class for all small positive $\epsilon$. We claim that $\hat{\mathcal{E}}$ is $\hat{\omega}_{\epsilon}$-stable for $\epsilon$ small enough. Indeed, suppose the contrary. Then there exists a sequence $\epsilon-k$ converging to 0 such that $\hat{\mathcal{E}}$ is not $\hat{\omega}_{\epsilon_{k}}$-stable. Let $\mathcal{S}_{i} \subset \hat{\mathcal{E}}$ be the maximal destabilizing subsheaf with respect to $\hat{\omega}_{\epsilon_{i}}$. Since $a(\hat{X})=0$, we find $i_{0}$ such that $\mathcal{S}_{i}=\mathcal{S}_{j}$ for all $i, j \geq i_{0}$ (Prop. 6.12), possibly after passing to a subsequence (but even this could be avoided). So let $\mathcal{S}=\mathcal{S}_{i}, i \geq i_{0}$. Then we have

$$
\frac{c_{1}(\mathcal{S}) \cdot \hat{\omega}_{\epsilon_{k}}^{n-1}}{s} \geq \frac{c_{1}(\hat{\mathcal{E}}) \cdot \hat{\omega}_{\epsilon_{k}}^{n-1}}{r},
$$

so passing to the limit,

$$
\frac{c_{1}(\mathcal{S}) \cdot \hat{\omega}^{n-1}}{s} \geq \frac{c_{1}(\hat{\mathcal{E}}) \cdot \omega^{n-1}}{r} .
$$

This contradicts $(+)$.

Thus $\hat{\mathcal{E}}$ is $\hat{\omega}$-stable for small positive $\epsilon$. Therefore $\hat{\mathcal{E}}$ is HermiteEinstein with respect to $\hat{\omega}_{\epsilon}$ and hence

$$
c_{1}^{2}(\hat{\mathcal{E}}) \cdot \hat{\omega}_{\epsilon}^{n-2} \leq \mu c_{2}(\hat{\mathcal{E}}) \cdot \hat{\omega}_{\epsilon}
$$


hence

$$
c_{1}^{2}(\hat{\mathcal{E}}) \cdot \hat{\omega}^{n-2} \leq \mu c_{2}(\hat{\mathcal{E}}) \cdot \hat{\omega}^{n-2} .
$$

Since $\operatorname{codim}(\operatorname{Sing}(X) \cup \operatorname{Sing}(\mathcal{E})) \geq 3$, we conclude

$$
c_{1}^{2}(\mathcal{E}) \omega^{n-2} \leq \mu c_{2}(\mathcal{E}) \cdot \omega^{n-2} .
$$

q.e.d.

Proposition 6.10. Let $L$ be a line bundle or a reflexive sheaf of rank 1 on the normal compact complex space $X$. Suppose $a(X)=0$. Let $\mathcal{S}_{i} \subset L$ be reflexive subsheaves, $i \in I$. Then for all $i$ there are only finitely many $j$ such that $\mathcal{S}_{j} \not \subset \mathcal{S}_{i}$.

Proof. Of course we may assume $X$ smooth. Since $a(X)=0$, the complex space $X$ has only finite many irreducible hypersurfaces $Y_{1}, \ldots Y_{r}$, therefore we can write

$$
\mathcal{S}_{i}=L-\sum_{j=1}^{r} a_{j}^{(i)} Y_{j}
$$

with $a_{j}^{(i)} \geq 0$. Thus the claim is clear.

q.e.d.

Definition 6.11. Let $\mathcal{F}$ be a torsion free coherent sheaf on a normal compact complex space and let $\mathcal{S} \subset \mathcal{F}$ be a reflexive subsheaf with $0<\mathrm{rkS}<\mathrm{rk} \mathcal{F}$. We say that $\mathcal{S}$ is maximal, if there is no proper reflexive subsheaf $\mathcal{S}^{\prime} \subset \mathcal{F}$ of the same rank as $\mathcal{S}$ such that $\mathcal{S} \subset \mathcal{S}^{\prime}$ and $\mathcal{S} \neq \mathcal{S}^{\prime}$

If $\omega$ is a Kähler form on $X$ and if $\mathcal{S}$ is the $\omega$-maximal destabilizing subsheaf of the $\omega$-unstable sheaf $\mathcal{F}$, then $\mathcal{S}$ is maximal. This is the way we will identify maximal subsheaves.

Proposition 6.12. Let $X$ be a normal compact Kähler space with $a(X)=0$ and $\mathcal{F}$ a reflexive coherent sheaf on $X$. Then $\mathcal{F}$ admits only finitely many maximal reflexive subsheaves of rank 1 .

Proof. Of course we may assume $X$ smooth. Consider now the maximal subsheaves $\mathcal{S}_{i} \subset \mathcal{F}$ of rank $1, i \in I=\mathbb{N}$. Choose $m \in \mathbb{N}$ and $i_{1}<\cdots<i_{m}$ such that

$$
\mathcal{S}^{\prime}=\mathcal{S}_{i_{1}}+\cdots+\mathcal{S}_{i_{m}} \subset \mathcal{F}
$$


has the following property: if $j$ is different from the indices $i_{1}, \ldots, i_{m}$, then $\operatorname{rk} \mathcal{S}^{\prime}=\operatorname{rk}\left(\mathcal{S}^{\prime}+\mathcal{S}_{j}\right)$. So things come down to show that there are only finitely many $j$ such that

$$
\operatorname{rk}\left(\mathcal{S}_{j} \cap \mathcal{S}^{\prime}\right)=1
$$

In order to prove this, we assume to the contrary that there are infinitely many $j$ such that $\operatorname{rk}\left(\mathcal{S}_{j} \cap \mathcal{S}^{\prime}\right)=1$. Then we have infinitely many subsheaves

$$
\mathcal{T}_{j}:=\mathcal{S}_{j} \cap \mathcal{S}^{\prime} \subset \mathcal{S}^{\prime}
$$

of rank 1 (use again the finiteness of hypersurfaces in $X$.) Now fix $j_{0}$. Then by (6.10) there are only finitely many $j$ such that $\mathcal{T}_{j} \not \subset \mathcal{T}_{j_{0}}$. For all others we have $\mathcal{T}_{j} \subset \mathcal{T}_{j_{0}}$ and for those we write

$$
\mathcal{T}_{j}=\mathcal{S}_{j}-A_{j}
$$

and

$$
\mathcal{T}_{j_{0}}=\mathcal{S}_{j_{0}}-A_{j_{0}}
$$

with effective divisors $A_{j}$. Since $X$ has only finitely many irreducible hypersurfaces, we have $A_{j_{0}} \subset A_{j}$ for almost all $j$, hence we obtain $\mathcal{S}_{j} \subset \mathcal{S}_{j_{0}}$ for almost all $j$, contradiction to maximality. $\quad$ q.e.d.

Corollary 6.13. Let $X$ be an normal compact Kähler space with $a(X)=0$ and $\mathcal{F}$ a torsion free sheaf of rank at most 3 . Then $\mathcal{F}$ contains only finitely many maximal reflexive subsheaves.

Proof. By 6.12 we have only to deal with the case of subsheaves of rank 2. This is done by dualizing and applying 6.12 to $\mathcal{F}^{*}$ using the following trivial remark: if $\mathcal{S} \subset \mathcal{F}$ is maximal with quotient $Q$, then $Q^{*} \subset \mathcal{F}^{*}$ is maximal.

q.e.d.

\section{An abundance theorem for Kähler threefolds}

Here we want to solve (the remaining part of) the abundance problem for Kähler threefolds:

Theorem 7.1. Let $X$ be $a \mathbb{Q}$-Gorenstein Kähler threefold with only terminal singularities such that $K_{X}$ is nef ( a minimal Kähler threefold for short). Then $\kappa(X) \geq 0$.

Of course, more should be true: 
Conjecture 7.2. Let $X$ be a minimal Kähler threefold. Then $K_{X}$ is semi-ample, i.e., some multiple $m K_{X}$ is spanned by global sections.

\section{Remark 7.3.}

(1) In case $X$ is projective, everything is proved by Miyaoka [23, 24] and Kawamata [18].

(2) In the non-algebraic case, 7.1/7.2 is proved in [27] with the important possible exception that $X$ is simple and not Kummer (see 1.4). In particular in this remaining case we have algebraic dimension $a(X)=0$ and $\pi_{1}(X)$ finite. In [9] 7.1 is proved if $K_{X}$ carries a sufficiently nice metric, e.g., if $K_{X}$ is hermitian semipositive.

(3) In case $X$ is Gorenstein, we have the Riemann-Roch formula

$$
\chi\left(X, \mathcal{O}_{X}\right)=-\frac{1}{24} K_{X} \cdot c_{2}(X)
$$

Therefore, if we recall that we may assume $a(X)=0$, the inequality $(6.1)$

$$
K_{X} \cdot c_{2}(X) \geq 0
$$

implies $\chi\left(X, \mathcal{O}_{X}\right) \leq 0$ and therefore $h^{0}\left(X, K_{X}\right)=h^{3}\left(X, \mathcal{O}_{X}\right) \neq 0$, so that at least $\kappa(X) \geq 0$. In case $X$ is not Gorenstein, this Riemann-Roch formula is not true; instead one has some positive correction term [12] which might correct the negativity of the product $-K_{X} \cdot c_{2}(X)$ and therefore destroy the contradiction.

Proof of Theorem 7.1. As noticed in 7.3 we may assume that $X$ is simple non-Kummer, in particular $q(X)=0$. First we reduce ourselves to the case that $X$ is $\mathbb{Q}$-factorial by applying Kawamata's factorialisation $f: \hat{X} \longrightarrow X$ as in the proof of 4.4. Since $f$ is small, we have $K_{\hat{X}}=$ $f^{*}\left(K_{X}\right)$, so $K_{\hat{X}}$ is nef. Hence we can work on $\hat{X}$ and thus may assume $X$ to be $\mathbb{Q}$-factorial from the beginning. We consider a desingularization

$$
\pi: \hat{X} \longrightarrow X
$$

and compute by Riemann-Roch

$$
\chi\left(\hat{X}, \pi^{*}\left(m K_{X}\right)\right)=\frac{m}{12} K_{X} \cdot c_{2}(X)+\chi\left(X, \mathcal{O}_{X}\right)
$$


for all $m$ such that $m K_{X}$ is Cartier. Assume $\kappa(X)=-\infty$, so $H^{3}\left(\mathcal{O}_{X}\right)=$ 0 . Since $X$ is not projective, we have $H^{2}\left(\mathcal{O}_{X}\right) \neq 0$. In total we obtain:

$$
\chi\left(X, \mathcal{O}_{X}\right) \geq 2
$$

If now $K_{X} \neq 0$, then by $4.3 / 5.1$, we have $h^{2}\left(X, m K_{X}\right) \leq 1$, hence $(6.1)$ and $(*)$ imply $h^{0}\left(X, m K_{X}\right) \geq 1$, a contradiction. If however $K_{X}=0$, take a positive integer $m$ such that $m K_{X}$ is Cartier. If now $m K_{X}$ is not a torsion line bundle, we must have $q(X)>0$, contradiction. q.e.d.

Remark 7.4. In order to settle the abundance for Kähler threefolds completely, it remains to show that a simple threefold $X$ with $K_{X}$ nef and $\kappa(X)=0$ must be Kummer. In the following we collect what we know about $X$. We shall assume that $q(X)=0$, otherwise we consider the Albanese and are easily done. Thus we have $\chi\left(X, \mathcal{O}_{X}\right) \geq 1$.

(1) $K_{X} \cdot c_{2}(X)=0$ and $1 \leq \chi\left(X, \mathcal{O}_{X}\right) \leq 2$.

The first part follows easily from equation $(*)$ in the proof of $(7.3)$ together with 4.1/5.1. Hence

$$
\chi\left(X, m K_{X}\right)=\chi\left(X, \mathcal{O}_{X}\right)
$$

for all integers $m$ such that $m K_{X}$ is Cartier. Then again $4.3 / 5.1$ gives the inequality for $\chi\left(X, \mathcal{O}_{X}\right)$.

(2) $X$ cannot be Gorenstein. In fact, then the Riemann-Roch formula

$$
24 \chi\left(X, \mathcal{O}_{X}\right)=-K_{X} \cdot c_{2}(X)=0
$$

gives a contradiction.

(3) If $\chi\left(X, \mathcal{O}_{X}\right)=2$, then $K_{X}^{2}=0$. This is a consequence of (1) via the vanishing 4.3 .

(4) If $\chi\left(X, \mathcal{O}_{X}\right)=1$, then $h^{0}\left(X, K_{X}\right)=1$ and $h^{2}\left(X, \mathcal{O}_{X}\right)=1$.

\section{Almost algebraic Kähler threefolds}

In this section we show that simple non-Kummer threefolds are very far from projective threefolds, in a sense which is made precise in the following definition: 
Definition 8.1. Let $X$ be a normal Kähler variety with only terminal singularities. $X$ is almost algebraic if there exists an algebraic approximation of $X$. This is a proper surjective flat holomorphic map $\pi: \mathcal{X} \rightarrow \Delta$ from a normal complex space $\mathcal{X}$ where $\Delta \subset \mathbb{C}^{m}$ is the unit disc, where $X \simeq X_{0}$, where all complex analytic fibers $X_{t}=\pi^{-1}(t)$ are normal Kähler spaces with at most terminal singularities such that there is a sequence $\left(t_{j}\right)$ in $\Delta$ converging to 0 so that all $X_{j}:=X_{t_{j}}$ are projective.

Of course, in case $X$ is smooth, all $X_{t}$ will be smooth (after possibly shrinking $\Delta$ ).

The following problem is attributed to Kodaira:

Problem 8.2. Is every compact Kähler manifold almost algebraic?

From a point of view of algebraic geometry almost algebraic Kähler spaces seem to be the most interesting Kähler spaces. Therefore it is worthwile to notice

Theorem 8.3. Let $X$ be a nearly algebraic Kähler threefold with only terminal singularities. If $X$ is simple and additionally $K_{X}$ nef or $X$ smooth, then $X$ is Kummer.

Proof. Assume that $X$ is not Kummer. Then $\pi_{1}(X)$ is finite by [3] as already mentioned. Let $\pi: \mathcal{X} \rightarrow \Delta$ be an algebraic approximation of $X$. Let $\left(t_{j}\right)$ be a sequence in $\Delta$ converging to 0 such that all $X_{j}=X_{t_{j}}$ are projective. Notice first that $\kappa\left(X_{j}\right) \geq 0$ for all $j$. In fact, otherwise $X_{j}$ would be uniruled for some $j$ and by standard arguments $X_{t}$ would be uniruled for all $t$ which is not possible, $X=X_{0}$ being simple.

(1) We show that $\kappa(X)=\kappa\left(X_{0}\right) \geq 0$. Fix a positive integer $m$. Then by $[21,1.6]$, every $t_{j}$ admits an open neighborhood $U_{j}$ such that

$$
h^{0}\left(X_{t}, m K_{X_{t}}\right)=h^{0}\left(X_{j}, m K_{X_{j}}\right)
$$

for all $t \in U_{j}$. Now choose $m$ such that $h^{0}\left(X_{j}, m K_{X_{j}}\right)>0$ for some $j$. Then it follows that $h^{0}\left(X_{t}, m K_{X_{t}}\right)=h^{0}\left(X_{j}, m K_{X_{j}}\right)=: d>0$ for all $t$ in an open set in $\Delta$. Let

$$
A:=\left\{t \in \Delta \mid h^{0}\left(X_{t}, m K_{X_{t}}\right) \geq d\right\} .
$$

Then $A$ is an analytic set in $\Delta$ (semi-continuity in the analytic Zariski topology), and it contains a nonempty open set, hence $A=$ $\Delta$. Thus $\kappa\left(X_{0}\right) \geq 0$. Since $X_{0}$ is simple, we conclude $\kappa\left(X_{0}\right)=0$. 
(2) Suppose that $\kappa\left(X_{j}\right) \geq 1$ for some $j$. Then fix $m$ such that $h^{0}\left(X_{j}, m K_{X_{j}}\right) \geq 2$. Repeating the same arguments as in (1), we conclude $h^{0}\left(X, m K_{X}\right) \geq 2$, contradicting $X$ being simple. So $\kappa\left(X_{j}\right)=0$ for all $j$.

(3) Here we will show that $X_{j}$ is Kummer for all $j$. Let $X_{j}^{\prime}$ be a minimal model of $X_{j}$. Observe that

$$
h^{2}\left(X_{j}, \mathcal{O}_{X_{j}}\right)=h^{2}\left(X, \mathcal{O}_{X}\right)>0
$$

in fact, $H^{1}\left(X, \mathcal{O}_{X}\right)=0$, hence $H^{1}\left(X_{j}, \mathcal{O}_{X_{j}}\right)=0$ for large $j$. Moreover $h^{0}\left(X_{t}, K_{X_{t}}\right)$ is constant by [KM92], as shown above. Therefore the equality follows by Serre duality and the constancy of $\chi\left(X_{t}, \mathcal{O}_{X_{t}}\right)$. Hence we also have $h^{2}\left(X_{j}^{\prime}, \mathcal{O}_{X_{j}^{\prime}}\right)>0$.

Since $K_{X_{j}^{\prime}} \equiv 0$, there exists a finite cover, the so-called canonical cover, $h: \widetilde{X}_{j} \rightarrow X_{j}^{\prime}$, étale in codimension 2 , such that $K_{\widetilde{X}_{j}}=\mathcal{O}_{\widetilde{X}_{j}}$. In particular $\widetilde{X}_{j}$ is Gorenstein and Riemann-Roch yields

$$
\chi\left(\widetilde{X}, \mathcal{O}_{\widetilde{X}_{j}}\right)=0
$$

Since $h^{2}\left(\mathcal{O}_{\widetilde{X}_{j}}\right)>0$, we must have $q\left(\widetilde{X}_{j}\right)>0$. Let $\alpha_{j}: \widetilde{X}_{j} \longrightarrow$ $A=A_{j}$ be the Albanese map. By [Ka85] there exists a finite étale cover $B \longrightarrow A$ such that

$$
\hat{X}_{j}:=\widetilde{X}_{j} \times_{A} B \simeq F \times B .
$$

In particular $\hat{X}_{j}$ and $\widetilde{X}_{j}$ are smooth because of the isolatedness of singularities. We conclude that $X_{j}^{\prime}$ is Kummer unless $F$ is a K3-surface. To exclude that case, consider the image $F^{\prime} \subset \tilde{X}_{j}^{\prime}$ of a general $F \times\{b\}$. Then $F^{\prime}$ is K3 or Enriques and does not meet the singularities of $X_{j}^{\prime}$. Moreover the normal bundle $N_{F^{\prime}}$ is numerically trivial. Since $F^{\prime}$ moves, it is actually trivial. Now consider the strict transform in $X_{j}$, again called $F^{\prime}$. Then $F^{\prime}$ has the same normal bundle in $X_{j}$, so that $N_{F^{\prime} / \mathcal{X}}=\mathcal{O}_{\mathcal{X}}^{2}$. Since $H^{1}(N)=0$, the deformations of $F^{\prime}$ cover every $X_{t}$ contradicting the simplicity of $X_{0}$. So $F$ cannot be K3 and $X_{j}$ is Kummer.

(4) Suppose $K_{X_{0}}$ nef. Fix a positive number $m$ such that

$$
m K_{\mathcal{X}}=\mathcal{O}_{\mathcal{X}}(D)
$$


with some effective divisor $D$. We may assume that $D$ does not contain any fiber of $\pi$; denote $D_{t}=X_{t} \cap D$. We want to argue that $K_{X_{t}}$ must be nef, therefore $K_{X_{j}}=0$ so that $D_{j}=0$ and $D=0$ in total. So we will obtain $K_{X}=0$. To see that $X$ is Kummer, consider the canonical cover of $X$ and argue as in the proof of (7.1). To prove nefness, we apply [KM92] to deduce that the sequence $\varphi_{j}: X_{j} \rightarrow X_{j}^{\prime}=A_{j} / G$ appears in family $\mathcal{X}_{U_{j}} \longrightarrow \mathcal{X}_{U_{j}}^{\prime}$ over a small neighborhood $U_{j}$ of $t_{j}$. In particular some multiple $N_{D_{t}}^{* \mu}$ has many sections for $t \in U_{j}$ on an at least 1-dimensional family of curves. By semicontinuity, also $N_{D_{0}}^{* \mu}$ has many sections on such a family, contradicting the nefness of $D_{0}$. Alternatively, $K_{\mathcal{X}}$ is negative on a family of rational curves over $U_{j}$, which converges to a family of rational curves in $X_{0}$ and therefore forces $K_{X_{0}}$ to be non-nef.

(5) Now suppose $X_{0}$ smooth, i.e., $\pi$ is smooth after shrinking $\Delta$. Take a sequence of blow-ups of smooth subvarieties of $\mathcal{X}$ such that the preimage of $\operatorname{red} D$ has normal crossings. After shrinking $\Delta$ we may assume that the only points and compact curves blown up lying over $X_{0}$ so that all fibers over $\Delta \backslash 0$ are smooth. Then take a covering $h: \widetilde{\mathcal{X}} \longrightarrow \mathcal{X}$ such that $K_{\widetilde{\mathcal{X}}}=\mathcal{O}(\widetilde{D})$ with $\widetilde{\mathcal{X}}$ smooth. This is possible e.g., by applying [Ka81]. Then $\mathcal{X}_{t_{j}}$ is Kummer and admits a 3 -form and therefore must be bimeromorphically a torus (if $A / G$ admits a 3 -form, then it is a torus covered by $A$. This is a consequence of the simplicity of $A$ and the fact that $G$ acts without fix points). Hence every $\widetilde{\mathcal{X}}_{t}, t \neq 0$, has 3 holomorphic 1-forms which are independent at the general point and therefore every $X_{t}, t \neq 0$, is Kummer. In order to show that $X_{0}$ is Kummer, consider the central fiber $\widetilde{\mathcal{X}}_{0}$ which contains the preimage of the strict transform $X_{0}^{\prime}$ of $X_{0}$. More precisely, we have

$$
\widetilde{\mathcal{X}}_{0}=X_{0}^{\prime}+\sum a_{i} E_{i}
$$

where the $E_{i}$ are smooth threefolds contracted to points or curves. By semi-continuity, $h^{2}\left(\mathcal{O}_{\widetilde{\mathcal{X}}_{0}}\right) \geq 3$. Now we check easily that

$$
H^{2}\left(\widetilde{\mathcal{X}}_{0}, \mathcal{O}_{\widetilde{\mathcal{X}}_{0}}\right)=H^{2}\left(X_{0}^{\prime}, \mathcal{O}_{X_{0}^{\prime}}\right),
$$

hence $X_{0}^{\prime}$ carries three 2 -forms coming from $\widetilde{\mathcal{X}}$. But then it is clear that also some of the holomorphic 1-forms on $\widetilde{\mathcal{X}}$ give nonzero 1 forms on $X_{0}^{\prime}$, since the 2 -forms are wegdge products of the 1 -forms. Hence $X_{0}^{\prime}$ is Kummer and so does $X_{0}$.

q.e.d. 
If Problem 8.2 has a positive answer in dimension 3, Theorem 8.3 excludes the existence of simple non-Kummer threefolds.

\section{References}

[1] W. Barth, C. Peters, A. van de Ven, Compact complex surfaces, Ergebnisse der Mathematik und ihrer Grenzgebiete, 4, Springer, Berlin, 1984, MR 86c:32026, Zbl 0718.14023.

[2] M. Beltrametti \& A.J. Sommese, The adjunction theory of complex projective varieties, de Gruyter Exp. in Math., 16, Walter de Gruyter \& Co., Berlin, 1995, MR 96f:14004, Zbl 0845.14003.

[3] F. Campana, Remarques sur le revêtement universel des variétés Kählériennes compacts, Bull. Soc. Math. France 122 (1994) 255-284, MR 95f:32036, Zbl 0810.32013.

[4] F. Campana \& Th. Peternell, The Kodaira dimension of Kummer threefolds, Bull. Soc. Math. France 129 (2001) 357-359, MR 2003b:32022, Zbl 1001.32009.

[5] J.-P. Demailly, Regularization of closed positive currents and intersection theory, J. Alg. Geom. 1 (1992) 361-409, MR 93e:32015, Zbl 0777.32016.

[6] J.-P. Demailly, Monge-Ampère operators, Lelong numbers and intersection theory, Complex Analysis and Geometry, Univ. Series in Math., edited by V. Ancona and A. Silva, Plenum Press, New-York, 1993, 115-193, MR 94k:32009, Zbl 0792.32006.

[7] J.-P. Demailly, A numerical criterion for very ample line bundles, J. Differential Geom. 37 (1993) 323-374, MR 94d:14007, Zbl 0783.32013.

[8] J.-P. Demailly, Th. Peternell \& M. Schneider, Compact complex manifolds with numerically effective tangent bundles, J. Alg. Geom. 3 (1994) 295-345, MR 95f:32037, Zbl 0827.14027.

[9] J.-P. Demailly, Th. Peternell \& M. Schneider, Pseudo-effective line bundles on compact Kähler manifolds, Internat. J. Math. 12 (2001) 689-741, MR 2003a:32032.

[10] D. Eisenbud, Commutative algebra, Graduate Texts in Math., 150, Springer, 1995, MR 97a:13001, Zbl 0819.13001.

[11] I. Enoki, Stability and negativity for tangent sheaves of minimal Kähler spaces, Geometry and analysis on manifolds (Katata/Kyoto, 1987), Lecture Notes in Math., 1339, Springer, Berlin, 1988, 118-127, MR 90a:32039, Zbl 0661.53051.

[12] A.R. Fletcher, Contributions to Riemann-Roch on projective 3-folds with only canonical singularities and applications, Algebraic geometry, Bowdoin, 1985 (Brunswick, Maine, 1985), Proc. Symp. Pure Math. 46, Part 1, Amer. Math. Soc., Providence, 1987, 221-231, MR 89h:14032, Zbl 0662.14026. 
[13] H. Grauert, Über Modifikationen und exzeptionelle analytische Mengen, Math. Ann. 146 (1962) 331-368, MR 25 \#583, Zbl 0173.33004.

[14] H. Grauert \& O. Riemenschneider, Verschwindungssätze für analytische Kohomologiegruppen auf komplexen Räumen, Invent. Math. 11 (1970) 263-292, MR 46 \#2081, Zbl 0202.07602.

[15] L. Hörmander, $L^{2}$ estimates and existence theorems for the $\bar{\partial}$ operator, Acta Math. 113 (1965) 89-152, MR 31 \#3691, Zbl 0158.11002.

[16] Y. Kawamata, Characterization of abelian varieties, Comp. Math. 43 (1981) 275-276, MR 83j:14029, Zbl 0471.14022.

[17] Y. Kawamata, Minimal models and the Kodaira dimension of algebraic fiber spaces, J. Reine Angew. Math. 363 (1985) 1-46, MR 87a:14013, Zbl 0589.14014.

[18] Y. Kawamata, Crepant blowing-up of 3-dimensional canonical singularities and its application to degenerations of surfaces, Ann. Math. 127 (1988) 93-163, MR 89d:14023, Zbl 0651.14005.

[19] Y. Kawamata, K. Matsuki \& K. Matsuda, Introduction to the minimal model problem, Algebraic geometry, Sendai, 1985, Adv. Stud. Pure Math., 10, NorthHolland, Amsterdam, 1987, 283-360, MR 89e:14015, Zbl 0672.14006.

[20] S. Kobayashi, Differential geometry of complex vector bundles, Publications of the Mathematical Society of Japan, 15, Kanô Memorial Lectures, 5, Princeton Univ. Press, Princeton, Iwanami Shoten, Tokyo, 1987, MR 89e:53100, Zbl 0708.53002.

[21] J. Kollár \& S. Mori, Classification of three-dimensional flips, J. Amer. Math. Soc. 5 (1992) 533-703, MR 93i:14015, Zbl 0773.14004.

[22] J. Kollaŕ, et al., Flips and abundance for algebraic threefolds, Papers from the Second Summer Seminar on Algebraic Geometry held at the University of Utah, Salt Lake City, Utah, August 1991, Astérisque, 211, Soc. Math. France, 1992, MR 94f:14013, MR 0782.00075.

[23] Y. Miyaoka, The Chern classes and Kodaira dimension of a minimal variety, Algebraic geometry, Sendai, 1985, Adv. Stud. Pure Math., 10, North-Holland, Amsterdam, 1987, 449-476, MR 89k:14022, Zbl 0648.14006.

[24] Y. Miyaoka, On the Kodaira dimension of minimal threefolds, Math. Ann. 281 (1988) 325-332, MR 89g:14028, Zbl 0625.14023.

[25] Y. Miyaoka, Abundance conjecture for 3-folds: case $\nu=1$, Comp. Math. 68 (1988) 203-220, MR 89m:14023, Zbl 0681.14019.

[26] M. Paun, Sur l'effectivité numérique des images inverses de fibrés en droites, Math. Ann. 310 (1998) 411-421, MR 99c:32042.

[27] Th. Peternell, Towards a Mori theory on compact Kähler 3-folds, III, Bull. Soc. Math. France 129 (2001) 339-356, MR 2003b:32024, MR 0994.32017. 
[28] M. Reid, Young person's guide to canonical singularities, Algebraic geometry, Bowdoin, 1985 (Brunswick, Maine, 1985), Proc. Symp. Pure Math., 46, Part 1, Amer. Math. Soc., Providence, 1987, 345-414, MR 89b:14016, Zbl 0634.14003.

[29] Y.T. Siu, Analyticity of sets associated to Lelong numbers and the extension of closed positive currents, Invent. Math. 27 (1974) 53-156, MR 50 \#5003, Zbl 0289.32003.

[30] H. Skoda, Sous-ensembles analytiques d'ordre fini ou infini dans $\mathbb{C}^{n}$, Bull. Soc. Math. France 100 (1972) 353-408, MR 50 \#5004, Zbl 0246.32009.

[31] J. Varouchas, Stabilité de la classe des variétés Kählériennes par certain morphismes propres, Invent. Math. 77 (1984) 117-127, MR 86a:32026, Zbl 0529.53049.

Université de Grenoble I, BP 74 Institut Fourier, UMR 5582 DU CNRS 38402 Saint-Martin D'Hères, France

UNIVERSITÄT BAYREUTH Mathematisches Institut D-95440 Bayreuth, Deutschland 